\title{
Multiple Spatio-Temporal Scale Modeling of Composites Subjected to Cyclic Loading
}

\author{
Robert Crouch $^{1}$, Caglar Oskay*1, and Stephen Clay² \\ ${ }^{1}$ Department of Civil and Environmental Engineering, \\ Vanderbilt University, Nashville, TN 37235 \\ ${ }^{2}$ Air Vehicles Directorate, Air Force Research Laboratory, \\ Wright-Patterson Air Force Base, OH 45433
}

\begin{abstract}
This manuscript presents a multiscale modeling methodology for failure analysis of composites subjected to cyclic loading conditions. Computational homogenization theory with multiple spatial and temporal scales is employed to devise the proposed methodology. Multiple spatial scales address the disparity between the length scale of material heterogeneities and the overall structure, whereas multiple temporal scales with almost periodic fields address the disparity between the load period and overall life under cyclic loading. The computational complexity of the multiscale modeling approach is reduced by employing a meso-mechanical model based on eigendeformation based homogenization with symmetric coefficients in the space domain, and an adaptive time stepping strategy based on a quadratic multistep method with error control in the time domain. The proposed methodology is employed to simulate the response of graphite fiber-reinforced epoxy composites. Model parameters are calibrated using a suite of experiments conducted on unidirectionally reinforced specimens subjected to monotonic and cyclic loading. The calibrated model is employed to predict damage progression in quasi-isotropic specimens. The capabilities of the model are validated using acoustic emission testing.
\end{abstract}

Keywords: Multiscale modeling; Computational homogenization; Composites; Fatigue; Damage mechanics.

\section{Introduction}

Composite materials provide tremendous potential as high performance structural components in aerospace applications and in other industries due to high specific strength, stiffness, and low weight. Composites also show good durability to cyclic loading with high fatigue limits. Despite favorable fatigue properties, the failure of composite structures is often sudden and catastrophic with few visible signatures. This leads to conservative designs strategies to contain failure events. Accurate modeling of failure processes in composite structures subjected to cyclic loading provides the potential to extend the applicability of composites in high performance applications.

A plethora of experimental investigations in the past few decades have shed light into the failure mechanisms in fiber reinforced composites subjected to cyclic loading (e.g., [1, 2], among many others). From the modeling perspective, continuum damage mechanics and fracture mechanics models are typically employed to describe failure under cyclic loading. Fracture mechanics based approaches rely

${ }^{*}$ Corresponding author. Address: VU Station B\#351831, 2301 Vanderbilt Place, Nashville, TN 37235, United States 
on incorporation of distinct cracks at the scale of the structure [3], at the scale of the constituents [4], or both [5]. A fracture mechanics based crack propagation criteria (e.g., Paris law) and a numerical methodology for crack propagation such as mesh refinement [6], virtual crack closure [7], cohesive zone [8], or the extended finite element method [9] are employed to describe fracture events within the composite material. In the continuum damage mechanics approach, failure is described as the initiation and growth of diffuse damage (e.g., microcrack density) typically represented using internal state variables. The evolution of diffuse damage as a function of loading history is modeled within the nonlinear, path-dependent constitutive modeling context by employing micromechanically-informed damage evolution models such as the critical element model [10,11] and others [12-14].

Modeling complex failure mechanisms and their interactions in composite structures subjected to cyclic loading is a multiscale problem in space and time. Multiple spatial scales exist since many failure mechanisms initiate and grow at the scale of the composite constituents defined by the representative volume of the composite, whereas the overall failure is assessed at the scale of the structure or structural component. Multiple temporal scales exist because of the disparity between the characteristic loading period, which may be on the order of seconds, and the overall life of the structure which may be on the order of years. The computational homogenization method $[15,16]$ based on mathematical homogenization theory [17-20] is a powerful multiscale modeling approach, which has been applied to nonlinear solid mechanics problems involving multiple spatial scales including the failure of composite materials.

Straightforward application of the computational homogenization-based modeling to evaluate the cyclic response of composite structures is prohibitive due to the tremendous computational cost associated with solving a two-scale nonlinear problem in space for large number of time steps necessary to evaluate life under cyclic loading. This difficulty is addressed using two approaches: (a) by introducing reduced order (meso-mechanical) models that can represent the small-scale response at a fraction of the cost without significantly compromising the solution accuracy; and, (b) by introducing cycle-stepping methodologies that eliminate the need to resolve to resolve each load cycle throughout the life of the structure or structural component. Reduced-order models based on transformation field analysis [21], proper orthogonal decomposition [22], eigenstrains [23], and others [24-26] have brought significant progress to reduced-order modeling in the presence of multiple spatial scales. More recently, Oskay and coworkers $[27,28]$ proposed a reduced order computational homogenization framework based on the eigendeformation idea which provides (a) the ability to model multiple failure mechanisms at the microstructure including matrix and fiber cracking, and interfacial debonding; and, (b) a hierarchy of reduced order models that can be adapted to meet accuracy needs. The tyranny of temporal scales is addressed by employing cycle-jump technique [29] or computational homogenization-based temporal multiscale modeling [30-32] that has been employed in the context of single spatial scale continuum damage mechanics and damage-plasticity models. More recently, Fish and coworkers applied cyclejump techniques to investigate the fatigue life of composites [33,34]. Despite progress, computational multiple spatio-temporal scale modeling for accurate, efficient and reliable prediction of failure in composite structures subjected to cyclic loading conditions remains to be a challenge.

In this manuscript, a new multiple spatio-temporal scale model for prediction of cyclic failure in composite materials is presented. The capabilities of the proposed model are demonstrated using a suite of experiments conducted on graphite fiber reinforced epoxy composites. The proposed model is devised using the computational homogenization theory with multiple spatial and temporal scales. The idea of almost periodicity of the response fields at the temporal scales [30] is employed to account for the presence of irreversible damage fields that violate the commonly assumed periodicity conditions of the response fields. The proposed model employs a reduced order modeling approach at the spatial domain using the eigendeformation based homogenization with symmetric coefficients, and an adaptive 

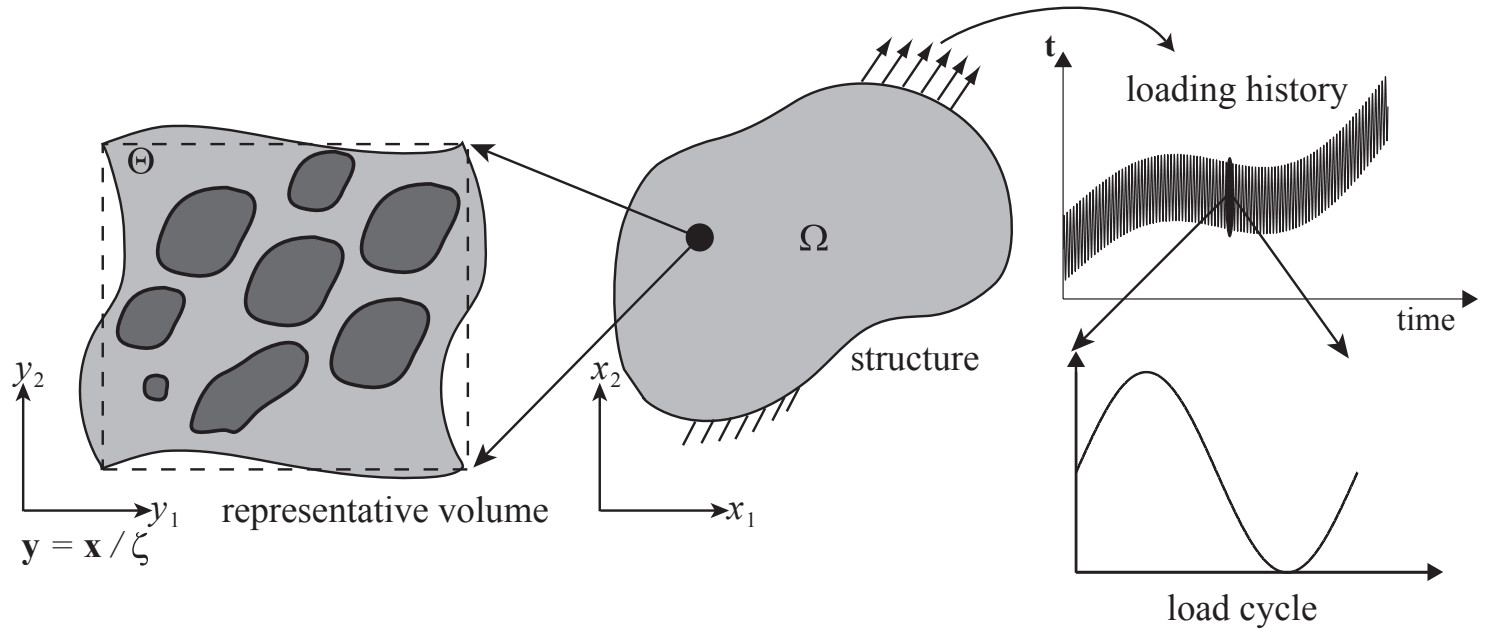

Figure 1: Multiple spatial and temporal scales.

time stepping strategy based on the modified multistep method to efficiently evaluate the response of a structural component by resolving only a fraction of the total number of cycles to failure. The proposed multiscale model is calibrated based on a suite of experiments conducted on graphite fiber reinforced epoxy (IM7/977-3) composite specimens under monotonic and cyclic loads, and validated against an independent set of experiments. The novel contributions of this manuscript are two-fold: To the best of the authors' knowledge, this manuscript is the first attempt to concurrently employ computational homogenization method with multiple temporal and spatial scales for failure modeling of heterogeneous materials subjected to cyclic loading. The second-order adaptive time stepping methodology proposed for adaptive error control in time provides improvements in accuracy compared to the first order time stepping approaches commonly employed in the context of cycle jump and temporal homogenization.

The remainder of this manuscript is organized as follows: Section 2 describes the multiple spatiotemporal problem setting. In Section 3, the computational approach used modeling cyclic failure behavior of composites is formulated. Section 4 and 5 provide the implementation details and the verification of the proposed modeling approach, respectively. Section 6 describes the experiments for calibration and validation of the computational approach, the calibration procedure of the material parameters based on the experimental data, and the validation of the model. Conclusions and future work are discussed in Section 7.

\section{Problem Statement}

We consider the progressive failure of a composite structure subjected to cyclic loading conditions. Let $\Omega \subset \mathbb{R}^{d}$ be the domain of a heterogeneous body, where $d=1,2$ or 3 denotes the number of space dimensions. $\Omega$ is composed of the repetition of a small periodic representative volume element (RVE), $\theta \subset \mathbb{R}^{d}$, composed of two or more distinct constituent materials as illustrated in Fig. 1. The governing 
equations describing the failure of the heterogeneous body are defined as ( $\mathbf{x} \in \Omega$ and $\left.t \in\left[0, t_{f}\right]\right)$ :

$$
\begin{gathered}
\nabla \cdot \boldsymbol{\sigma}^{\zeta \eta}(\mathbf{x}, t)+\mathbf{b}^{\zeta}(\mathbf{x})=0 \\
\boldsymbol{\sigma}^{\zeta \eta}(\mathbf{x}, t)=\left[1-\omega^{\zeta \eta}(\mathbf{x}, t)\right] \mathbf{L}^{\zeta}(\mathbf{x}): \boldsymbol{\epsilon}^{\zeta \eta}(\mathbf{x}, t)=\mathbf{L}^{\zeta}(\mathbf{x}):\left[\boldsymbol{\epsilon}^{\zeta \eta}(\mathbf{x}, t)-\boldsymbol{\mu}^{\zeta \eta}(\mathbf{x}, t)\right] \\
\boldsymbol{\epsilon}^{\zeta \eta}(\mathbf{x}, t)=\nabla^{s} \mathbf{u}^{\zeta \eta}(\mathbf{x}, t) \\
\dot{\omega}^{\zeta \eta}(\mathbf{x}, t)=f^{\zeta \eta}\left(\boldsymbol{\sigma}^{\zeta \eta}, \boldsymbol{\epsilon}^{\zeta \eta}, \mathbf{s}^{\zeta \eta}\right)
\end{gathered}
$$

where, $\mathbf{u}^{\zeta \eta}$ denotes displacement field; $\sigma^{\zeta \eta}$ the Cauchy stress; $\epsilon^{\zeta \eta}$ the total strain; $\omega^{\zeta \eta} \in[0,1)$ the scalar damage variable; $\boldsymbol{\mu}^{\zeta \eta}=\omega^{\zeta \eta} \epsilon^{\zeta \eta}$ the inelastic strain tensor; $\mathbf{b}^{\zeta}$ the body force; and $\mathbf{L}^{\zeta}$ the tensor of elastic moduli obeying the conditions of symmetry and positivity. The evolution of $\omega^{\zeta \eta}$ is typically nonlinear and history-dependent, and is provided in the functional form as a function of strain, stress and additional state variables, $\mathbf{s}^{\zeta \eta}$. A superposed dot denotes the material time derivative; $\mathbf{x}$ the position vector parameterizing the domain of the structure; $t$ the time coordinate; and, $\nabla \cdot(\cdot), \nabla(\cdot)$ and $\nabla^{s}(\cdot)$ the divergence, gradient and symmetric gradient operators, respectively. The boundary conditions prescribed on the body consist of slowly varying and oscillating components as illustrated in Fig. 1.

$$
\begin{aligned}
\mathbf{u}_{i}^{\zeta \eta}(\mathbf{x}, t) & =\hat{\mathbf{u}}^{\eta}(\mathbf{x}, t) ; \quad \mathbf{x} \in \Gamma_{u} ; \quad t \in\left[0, t_{\mathrm{o}}\right] \\
\boldsymbol{\sigma}^{\zeta \eta} \cdot \mathbf{n} & =\hat{\mathbf{t}}^{\eta}(\mathbf{x}, t) ; \quad \mathbf{x} \in \Gamma_{t} ; \quad t \in\left[0, t_{\mathrm{o}}\right]
\end{aligned}
$$

where, $\hat{\mathbf{u}}$ and $\hat{\mathbf{t}}$ are the prescribed displacements and tractions on the boundaries $\Gamma_{u}$ and $\Gamma_{t}$, respectively ( $\Gamma=\Gamma_{u} \cup \Gamma_{t}$ and $\Gamma_{u} \cap \Gamma_{t}=\emptyset$ ); and, $\mathbf{n}$ is the unit normal to $\Gamma_{t}$. The period of oscillations is taken to be slow enough that inertial forces are insignificant and the response remains quasi-static. The superscripts $\zeta$ and $\eta$ indicate that the response fields fluctuate in space and time, respectively. Double superscript indicates a response field that fluctuates in both time and space. The spatial fluctuations arise due to the fluctuating material properties within the RVE, whereas temporal fluctuations are due to the fast oscillatory component of the loading. The fluctuating spatio-temporal response is represented by introducing microscopic and microchronological scales parameterized by $\mathbf{y}=\mathbf{x} / \zeta$ and $\tau=t / \eta$, respectively; and, $0<\zeta \ll 1$ and $0<\eta \ll 1$ are scaling parameters. The original response fields that fluctuate in space and time are expressed as:

$$
\phi^{\zeta \eta}(\mathbf{x}, t)=\phi(\mathbf{x}, \mathbf{y}(\mathbf{x}), t, \tau(t))
$$

where, $\phi$ denotes an arbitrary response field. The macroscopic spatial derivatives of a response field are obtained through the chain rule:

$$
\nabla \phi^{\zeta}(\mathbf{x})=\nabla_{\mathbf{x}} \phi(\mathbf{x}, \mathbf{y})+\frac{1}{\zeta} \nabla_{\mathbf{y}} \phi(\mathbf{x}, \mathbf{y})
$$

in which, $\nabla_{\mathbf{x}}(\cdot)$ and $\nabla_{\mathbf{y}}(\cdot)$ are gradient operators with respect to macroscopic and microscopic coordinates, respectively. All response fields are assumed to be locally periodic with respect to the microscopic coordinates within the RVE throughout the deformation process: $\phi(\mathbf{x}, \mathbf{y})=\phi(\mathbf{x}, \mathbf{y}+\mathbf{k} \hat{\mathbf{y}})$, where, $\hat{\mathbf{y}}$ denotes the periods of the microstructure; and $\mathbf{k}$ is a $d \times d$ diagonal matrix with integer components. We consider the following spatial homogenization operator:

$$
\bar{\phi} \equiv\langle\phi\rangle_{\mathbf{y}}=\frac{1}{|\Theta|} \int_{\Theta} \phi d \mathbf{y}
$$

where, $|\Theta|$ denotes the volume of the RVE. 
The macrochronological derivative of a response field is expressed using the chain rule as:

$$
\dot{\phi}^{\eta}(t)=\dot{\phi}(t, \tau)=\phi_{, t}(t, \tau)+\frac{1}{\eta} \phi_{, \tau}(t, \tau)
$$

where, a comma followed by a subscript variable $t$ and $\tau$ denotes the partial time derivative with respect to the macrochronological and microchronological coordinates, respectively. In contrast to spatial variability, local periodicity is not a valid assumption for the response fields that vary in time. This is due to the presence of irreversible mechanisms associated with damage accumulation during a load cycle. The response fields are therefore assumed to be almost periodic, which implies that at neighboring points in a temporal domain homologous by the load period, the change in the value of a response function is small but does not vanish [31, 32]. Let:

$$
\langle\phi\rangle_{\tau}=\frac{1}{\tau_{0}} \int_{0}^{\tau_{0}} \phi(\mathbf{x}, \mathbf{y}, t, \tau) d \tau
$$

denote the temporal averaging operator; and, $\tau_{0}$ denote the period of scaled cyclic load $\left(\tau \in\left[0, \tau_{0}\right]\right)$. In the rate form, the almost periodic temporal homogenization operator is [30]:

$$
\dot{\tilde{\phi}}(t) \equiv \mathcal{M}(\phi)_{, t}=\tilde{\phi}_{, t}(t)+\phi_{\mathrm{ap}}(t)
$$

which satisfies the weak convergence property with respect to an arbitrary homogenization operator, $(\tilde{\cdot})$; and, $\phi_{\mathrm{ap}}=\left\langle\phi_{, \tau}\right\rangle_{\tau} / \eta$. Following the ideas of spatial homogenization theory, the natural choice for the temporal homogenization operator is the temporal averaging operator provided in Eq. 11. From the computational perspective, it is more convenient to choose a fixed-point operator that has the distributive property (i.e., $\phi=\psi \xi \rightarrow \tilde{\phi}=\tilde{\psi} \xi$ ) as evidenced by the ensuing formulation. In this study, $\tilde{\phi}(\mathbf{x}, \mathbf{y}, t)=\phi(\mathbf{x}, \mathbf{y}, t, 0)$ is adopted.

\section{Computational Model}

This section describes the multiscale spatio-temporal modeling approach to evaluate the failure response of heterogeneous bodies subjected to cyclic loading governed by Eqs. 1-6. The eigendeformationbased homogenization method with symmetric coefficients [28] is employed to address the multiple spatial scales, whereas temporal homogenization with almost periodic fields is employed to efficiently predict the life of a structure without resorting to full cycle-by-cycle analysis. The cyclic damage evolution law employed to idealize the failure response of composite constituents is presented.

\subsection{Multiple Scale Model}

We start by expressing the displacement field of the heterogeneous body using a two-scale asymptotic expansion:

$$
\mathbf{u}(\mathbf{x}, \mathbf{y}, t, \tau)=\overline{\mathbf{u}}(\mathbf{x}, t, \tau)+\zeta \mathbf{u}^{1}(\mathbf{x}, \mathbf{y}, t, \tau)
$$

in which, $\overline{\mathbf{u}}$ and $\mathbf{u}$ are the macroscopic and microscopic displacement fields, respectively. The governing equations are decomposed into macroscopic and microscopic problems through asymptotic analysis of the governing equations, which consists of substituting Eq. 13 into Eqs. 1-6, and collecting the same order terms of the resulting decompositions. The two leading order equilibrium equations are:

$$
\begin{array}{rll}
O\left(\zeta^{-1}\right) & : & \nabla_{\mathbf{y}} \cdot \boldsymbol{\sigma}(\mathbf{x}, \mathbf{y}, t, \tau)=0 \\
O(1) & : & \nabla_{\mathbf{x}} \cdot \boldsymbol{\sigma}(\mathbf{x}, \mathbf{y}, t, \tau)+\nabla_{\mathbf{y}} \cdot \boldsymbol{\sigma}^{1}(\mathbf{x}, \mathbf{y}, t, \tau)+\mathbf{b}(\mathbf{x}, \mathbf{y})=0
\end{array}
$$


where, $\nabla_{\mathbf{x}} \cdot(\cdot)$ and $\nabla_{\mathbf{y}} \cdot(\cdot)$ are divergence operators with respect to macroscopic and microscopic spatial coordinates, respectively; and, the body forces are assumed to remain constant in time for simplicity. The first and second order stress fields are:

$$
\begin{aligned}
\boldsymbol{\sigma}(\mathbf{x}, \mathbf{y}, t, \tau) & =[1-\omega(\mathbf{x}, \mathbf{y}, t, \tau)] \mathbf{L}(\mathbf{y}):\left[\overline{\boldsymbol{\epsilon}}(\mathbf{x}, t, \tau)+\nabla_{\mathbf{y}}^{s}\left(\mathbf{u}^{1}\right)(\mathbf{x}, \mathbf{y}, t, \tau)\right] \\
\boldsymbol{\sigma}^{1}(\mathbf{x}, \mathbf{y}, t, \tau) & =[1-\omega(\mathbf{x}, \mathbf{y}, t, \tau)] \mathbf{L}(\mathbf{y}):\left[\nabla_{\mathbf{x}}^{s}\left(\mathbf{u}^{1}\right)(\mathbf{x}, \mathbf{y}, t, \tau)\right]
\end{aligned}
$$

in which, $\nabla_{\mathbf{x}}^{s}(\cdot)$ and $\nabla_{\mathbf{y}}^{s}(\cdot)$ are symmetric gradient operators with respect to the macroscopic and microscopic spatial coordinates, respectively; and, $\overline{\boldsymbol{\epsilon}}=\nabla_{\mathbf{x}}^{s}(\overline{\mathbf{u}})$ is the macroscopic strain tensor.

Applying Eq. 10 to the damage evolution equations and collecting the same order terms yield:

$$
\begin{aligned}
& O\left(\eta^{-1}\right): \omega_{, \tau}=f^{0}(\boldsymbol{\sigma}, \boldsymbol{\epsilon}, \mathbf{s}) \\
& O(1): \omega_{, t}=f^{1}(\boldsymbol{\sigma}, \boldsymbol{\epsilon}, \mathbf{s})
\end{aligned}
$$

where, the evolution functions $f^{0}$ and $f^{1}$ are derived based on the prescribed evolution law $f$.

The boundary data applied to the heterogeneous body is composed of a slowly varying and a periodic oscillatory component:

$$
\begin{aligned}
\hat{\mathbf{u}}^{\eta}(\mathbf{x}, t) & =\hat{\mathbf{u}}^{0}(\mathbf{x}, t)+\hat{\mathbf{u}}^{1}(\mathbf{x}, \tau) \\
\hat{\mathbf{t}}^{\eta}(\mathbf{x}, t) & =\hat{\mathbf{t}}^{0}(\mathbf{x}, t)+\hat{\mathbf{t}}^{1}(\mathbf{x}, \tau)
\end{aligned}
$$

Applying spatial averaging (Eq. 9) to the $O(1)$ equilibrium equation (Eq. 15), exploiting the local periodicity of the stress fields in space, and applying the almost periodic temporal homogenization operator yields the macroscale equilibrium equation. The resulting equilibrium equation along with the spatio-temporally homogenized first order stress field, and the boundary conditions provide the macrochronological-macroscopic boundary value problem.

Macrochronological - Macroscopic Problem: Given: average body force, $\overline{\mathbf{b}}$, boundary data $\hat{\mathbf{u}}^{0}$ and $\hat{\mathbf{t}}^{0}$, and the solution of the macrochronological-microscopic problem; Find the macroscopic displacement field, $\tilde{\tilde{\mathbf{u}}}$, such that $\left(t \in\left[0, t_{f}\right]\right)$ :

$$
\begin{gathered}
\nabla_{\mathbf{x}} \cdot \tilde{\tilde{\boldsymbol{\sigma}}}(\mathbf{x}, t)+\overline{\mathbf{b}}(\mathbf{x})=\mathbf{0} ; \quad \mathbf{x} \in \Omega \\
\tilde{\tilde{\boldsymbol{\sigma}}}(\mathbf{x}, t)=\left\langle(1-\tilde{\boldsymbol{\omega}}) \mathbf{L}(\mathbf{y}):\left[\tilde{\tilde{\boldsymbol{\epsilon}}}+\nabla_{\mathbf{y}}^{s}\left(\tilde{\mathbf{u}}^{1}\right)\right]\right\rangle_{\mathbf{y}} ; \quad \mathbf{x} \in \Omega \\
\tilde{\tilde{\mathbf{u}}}=\hat{\mathbf{u}}^{0}(\mathbf{x}, t) ; \quad \mathbf{x} \in \Gamma_{u} \\
\tilde{\tilde{\boldsymbol{\sigma}}}(\mathbf{x}, t) \cdot \mathbf{n}=\hat{\mathbf{t}}^{0}(\mathbf{x}, t) ; \quad \mathbf{x} \in \Gamma_{t}
\end{gathered}
$$

Applying the temporal homogenization operator to the $O\left(\zeta^{-1}\right)$ equilibrium equations (Eq. 14) along with the constitutive equation for the leading order stress (Eq. 16), and employing periodic boundary conditions for the microscale displacement field, the macrochronological-microscopic boundary value problem is obtained.

Macrochronological-Microscopic Problem: At a fixed macroscale material point $\breve{\mathbf{x}} \in \Omega$; Given: macroscale strain, $\tilde{\tilde{\epsilon}}$, and the tensor of elastic moduli, L; Find the displacement field, $\tilde{\mathbf{u}}^{1}$, such that:

$$
\begin{aligned}
& \nabla_{\mathbf{y}} \cdot\left\{(1-\tilde{\omega}) \mathbf{L}(\mathbf{y}):\left[\tilde{\overline{\boldsymbol{\epsilon}}}+\nabla_{\mathbf{y}}^{s}\left(\tilde{\mathbf{u}}^{1}\right)\right]\right\}=\mathbf{0} ; \quad \mathbf{y} \in \theta \\
& \dot{\tilde{\omega}}(\breve{\mathbf{x}}, t)=f^{1}(\tilde{\boldsymbol{\sigma}}, \tilde{\boldsymbol{\epsilon}}, \tilde{\mathbf{s}})+\omega_{\mathrm{ap}}(\breve{\mathbf{x}}, t) ; \quad \mathbf{y} \in \theta \\
& \tilde{\mathbf{u}}^{1} \text { periodic on } \mathbf{y} \in \partial \theta
\end{aligned}
$$


The macro- and microscopic problems associated with the fast time scale at a fixed slow time coordinate, $t$, are obtained based on similar algebra, but without applying the temporal homogenization operator to the governing equations and considering the damage evolution equation with respect to the fast time scale (i.e., Eq. 18). The resulting microchronological - macroscopic and microchronological - microscopic problems are stated as follows:

Microchronological - Macroscopic Problem: At a fixed macrochronological time $\breve{t} \in\left[0, t_{f}\right]$, Given: average body force, $\overline{\mathbf{b}}$, boundary data, $\hat{\mathbf{u}}$ and $\hat{\mathbf{t}}$, and the solution of the microchronological-microscopic problem; Find the macroscopic displacement field, $\overline{\mathbf{u}}$, such that $\left(\tau \in\left[0, \tau_{0}\right]\right)$ :

$$
\begin{gathered}
\nabla_{\mathbf{x}} \cdot \overline{\boldsymbol{\sigma}}(\mathbf{x}, \breve{t}, \tau)+\overline{\mathbf{b}}(\mathbf{x})=\mathbf{0} ; \quad \mathbf{x} \in \Omega \\
\overline{\boldsymbol{\sigma}}=\left\langle(1-\omega) \mathbf{L}(\mathbf{y}):\left[\overline{\boldsymbol{\epsilon}}+\nabla_{\mathbf{y}}^{s}\left(\mathbf{u}^{1}\right)\right]\right\rangle_{\mathbf{y}} ; \quad \mathbf{x} \in \Omega \\
\overline{\mathbf{u}}=\hat{\mathbf{u}}(\mathbf{x}, \breve{t}, \tau) ; \quad \mathbf{x} \in \Gamma_{u} \\
\overline{\boldsymbol{\sigma}} \cdot \mathbf{n}=\hat{\mathbf{t}}(\mathbf{x}, \breve{t}, \tau) ; \quad \mathbf{x} \in \Gamma_{t}
\end{gathered}
$$

Microchronological-Microscopic Problem: At a fixed macroscale material point $\breve{\mathbf{x}} \in \Omega$ and a fixed macrochronological time $\breve{t} \in\left[0, t_{f}\right]$; Given: macroscopic strain, $\bar{\epsilon}$, and the tensor of elastic moduli, $\mathbf{L}$; Find the microscopic displacement field, $\mathbf{u}^{1}$, such that:

$$
\begin{gathered}
\nabla_{\mathbf{y}} \cdot\left\{(1-\omega) \mathbf{L}(\mathbf{y}):\left[\overline{\boldsymbol{\epsilon}}+\nabla_{\mathbf{y}}^{s}\left(\mathbf{u}^{1}\right)\right]\right\}=\mathbf{0} ; \quad \mathbf{y} \in \theta \\
\omega_{, \tau}(\breve{\mathbf{x}}, \mathbf{y}, \breve{t}, \tau)=f^{0}(\boldsymbol{\sigma}, \boldsymbol{\epsilon}, \mathbf{s}) ; \quad \mathbf{y} \in \theta \\
\mathbf{u}^{1} \text { periodic on } \mathbf{y} \in \partial \theta
\end{gathered}
$$

The macrochronological and microchronological problems are coupled through the almost periodic rate operator that defines the evolution of the temporally homogenized response fields (i.e., Eq. 23b). Therefore, the evolution of the macrochronological fields at each macrochronological time coordinate requires the solution of the microchronological problem associated with that time coordinate. The macroscale problems at the macrochronological and microchronological time scales are coupled with the respective microscale problems through the constitutive relationship (Eq. 24b). The evaluation of the macroscopic stress at each macroscopic material point requires the solution of the microscopic RVE problem associated with that material point. When the finite element method is employed to evaluate the macroscale problem, a nonlinear microscale problem must be evaluated to update the stress at each integration point for each increment and iteration of every time step of the loading history. This is a significant computational burden.

\subsubsection{Reduced Order Spatial Homogenization}

We employ the eigendeformation-based reduced order homogenization method with symmetric coefficients (sEHM) to reduce the computational cost associated with evaluating the coupled nonlinear micro- and macroscopic problems. sEHM is introduced in Ref. [28] and a brief summary is provided herein. The premise of sEHM is to devise a low-cost approximation to the nonlinear microscale boundary value problem defined over the representative volume elements based on the idea of precomputing certain microstructural information (e.g., concentration tensors, localization operators, influence functions) through linear elastic simulations prior to the analysis of the macroscale structure.

The microscale displacement field is expressed as:

$$
\mathbf{u}^{1}(\mathbf{x}, \mathbf{y}, t, \tau)=\mathbf{H}(\mathbf{y}): \overline{\boldsymbol{\epsilon}}(\mathbf{x}, t, \tau)+\int_{\Theta} \mathbf{h}(\mathbf{y}, \hat{\mathbf{y}}): \boldsymbol{\mu}(\mathbf{x}, \hat{\mathbf{y}}, t, \tau) d \hat{\mathbf{y}}
$$


in which, $\mathbf{H}$ is the elastic influence function (a third-order tensor) obtained by substituting Eq. 26 into the microscale problem, and evaluating the microscale problem in the absence of damage; and $\mathbf{h}$ is the phase damage induced influence function provided by the particular solutions to the RVE problems obtained by substituting Eq. 26 into the microscale problem, and solving the microscale problem in the presence of phase damage (i.e., $\boldsymbol{\mu}$ ). The governing equations and the discrete approximations of the elastic and phase damage induced influence functions are provided in Ref. [27]. Meso-mechanical shape functions are employed to discretize damage and damage induced inelastic strain fields:

$$
\{\omega, \boldsymbol{\mu}\}(\mathbf{x}, \mathbf{y}, t)=\sum_{\gamma=1}^{n} N^{(\gamma)}(\mathbf{y})\left\{\omega^{(\gamma)}, \boldsymbol{\mu}^{(\gamma)}\right\}(\mathbf{x}, t, \tau)
$$

in which, $N^{(\gamma)}$ are the phase shape functions. We consider a partitioning of the RVE domain into $n$ non-overlapping subdomains, $\theta^{(\gamma)}$, such that $\theta^{(\gamma)} \cap \theta^{(\Delta)}=\emptyset$ if $\gamma \neq \Delta$. The phase shape functions are taken to be piecewise constant functions forming a partition of unity within the RVE:

$$
N_{\mathrm{ph}}^{(\gamma)}(\mathbf{y})=\left\{\begin{array}{l}
1 \text { if } \mathbf{y} \in \theta^{(\gamma)} \\
0 \text { elsewhere }
\end{array}\right.
$$

and, $\omega^{(\gamma)}$ and $\boldsymbol{\mu}^{(\gamma)}$ are damage variable and inelastic strains averaged over the partition, $\boldsymbol{\theta}^{(\gamma)}$. Substituting Eqs. 26-28 into the microscale problem (Eqs. 25), and considering variational arguments (see Ref. [28]), the governing equation of the microscale problem is reduced to the following algebraic form:

$$
\sum_{\Delta=1}^{n}\left\{\left[1-\omega^{(\Delta)}\right]\left[\hat{\mathbf{A}}^{(\alpha \Delta)}: \overline{\boldsymbol{\epsilon}}+\sum_{\gamma=1}^{n} \hat{\mathbf{B}}^{(\alpha \Delta \gamma)}: \boldsymbol{\mu}^{(\gamma)}\right]\right\}=\mathbf{0} \quad \forall \alpha=1,2, \ldots, n
$$

in which, $\hat{\mathbf{A}}^{(\alpha \Delta)}$ and $\hat{\mathbf{B}}^{(\alpha \Delta \gamma)}$ are coefficient tensors computed as a function of the influence functions, $\mathbf{H}$ and $\mathbf{h}$ as well as the elastic properties, $\mathbf{L}$. The macroscopic stress tensor is expressed in terms of the partition average damage variable and inelastic strains as:

$$
\overline{\boldsymbol{\sigma}}=\sum_{\Delta=1}^{n}\left[1-\omega^{(\Delta)}\right]\left[\overline{\mathbf{L}}^{(\Delta)}: \overline{\boldsymbol{\epsilon}}+\sum_{\alpha=1}^{n} \overline{\mathbf{P}}^{(\Delta \alpha)}: \boldsymbol{\mu}^{(\alpha)}\right]
$$

in which, $\overline{\mathbf{L}}^{(\Delta)}$ and $\overline{\mathbf{P}}^{(\Delta \alpha)}$ are coefficient tensors. The expressions for the coefficient tensors are provided in Ref. [28]. The macrochronological counterparts of the reduced order microscopic equilibrium and macroscopic stress are obtained by applying the almost periodic temporal homogenization operator to Eqs. 29 and 30.

\subsection{Cyclic Damage Model}

Continuum damage mechanics (CDM) is employed to describe the evolution of damage within a phase partition (i.e., $\omega^{(\gamma)}$ ). In contrast to monotonic CDM models, the evolution law is allowed to accumulate damage at subcritical loading levels to permit sensitivity to cyclic loading. Such a model previously employed in Refs. [30, 33], is adopted in this study:

$$
\dot{\omega}^{(\gamma)}=g \frac{d \Phi\left(v^{(\gamma)}\right)}{d v^{(\gamma)}}\left\langle\dot{v}^{(\gamma)}\right\rangle_{+} \quad \text { where } \quad 0 \leq g=\frac{\Phi\left(v^{(\gamma)}\right)}{\omega^{(\gamma)}} \leq 1 ; \quad \gamma=1,2, \ldots, n
$$

where, $p$ is the cyclic damage sensitivity parameter; $\langle\cdot\rangle_{+}$denotes MacCauley brackets; $\Phi$ the damage evolution law under monotonically increasing loads; and $v^{(\gamma)}$ the damage equivalent strain:

$$
v^{(\gamma)}=\sqrt{\frac{1}{2} \epsilon^{(\gamma)}: \mathbf{L}^{(\gamma)}: \boldsymbol{\epsilon}^{(\gamma)}}
$$




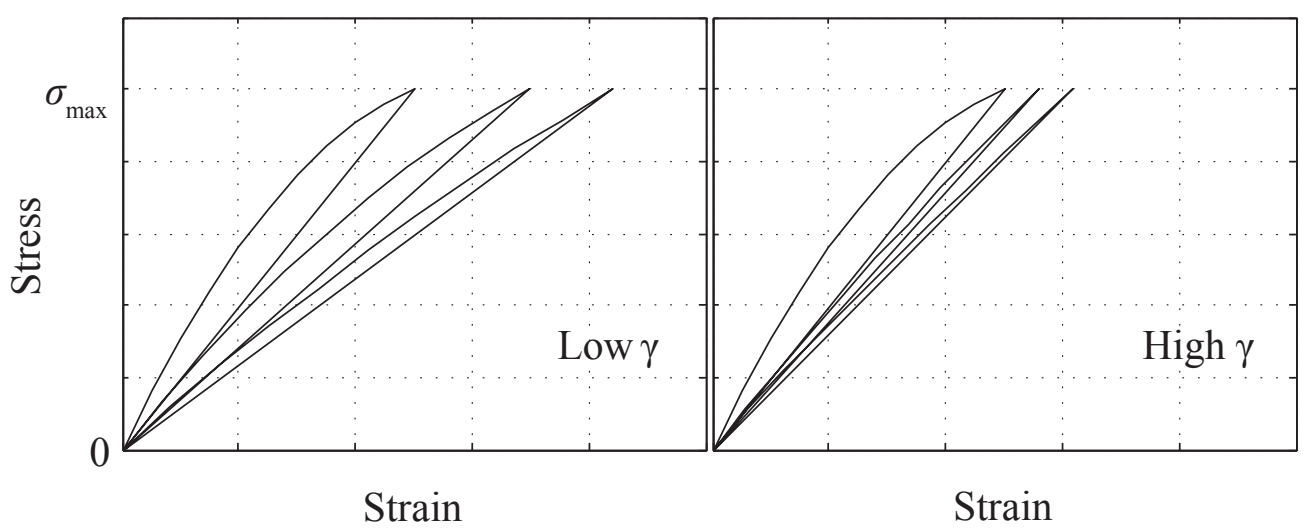

Figure 2: Effect of the cyclic damage sensitivity parameter, $p$, on the cyclic stress-strain relationship.

in which, $\mathbf{L}^{(\gamma)}$ is the tensor of elastic moduli of the constituent occupying $\theta^{(\gamma)}$; and, $\boldsymbol{\epsilon}^{(\gamma)}$ is the average strain within partition, $\gamma$. The damage evolution under monotonic conditions is idealized based on a smooth evolution law:

$$
\Phi\left(v^{(\gamma)}\right)=\frac{\arctan \left(a^{(\gamma)} v^{(\gamma)}-b^{(\gamma)}\right)+\arctan \left(b^{(\gamma)}\right)}{\frac{\pi}{2}+\arctan \left(b^{(\gamma)}\right)}
$$

where, $a^{(\gamma)}$ and $b^{(\gamma)}$ are material parameters associated with the constituent occupying domain $\theta^{(\gamma)}$.

The cyclic damage sensitivity parameter, $p$, which controls the rate of damage accumulation with respect to a load cycle as illustrated in Fig. 2, is taken to be of the form:

$$
p^{(\gamma)}=c_{0}^{(\gamma)}+c_{1}^{(\gamma)} v_{\max }^{(\gamma)}+c_{2}^{(\gamma)}\left(v_{\max }^{(\gamma)}\right)^{2}
$$

$c_{0}^{(\gamma)}, c_{1}^{(\gamma)}$, and $c_{2}^{(\gamma)}$ are parameters that account for the sensitivity of fatigue strength to the maximum applied loading. A quadratic form is chosen to model the fatigue behavior observed in the experiments. $v_{\max }^{(\gamma)}$ is the maximum value of the damage equivalent strain during the history of the material point as defined below.

$$
v_{\max }^{(\gamma)}(t)=\max _{T}\left\{v^{(\gamma)}(T) \mid 0 \leq T \leq t\right\}
$$

Applying the chain rule in the time domain (Eq. 10) to the damage evolution equation (Eq. 31), collecting the terms based on the order of the temporal scaling parameter, $\eta$, and applying the temporal homogenization operator to the $O(1)$ equation yields:

$$
\begin{aligned}
& \omega_{, \tau}^{(\gamma)}=f^{0}=g^{p} \frac{d \Phi\left(v^{(\gamma)}\right)}{d v^{(\gamma)}}\left\langle v_{, \tau}^{(\gamma)}\right\rangle_{+} \\
& \tilde{\omega}_{, t}^{(\gamma)}=f^{1}=g^{p} \frac{d \Phi\left(\tilde{v}^{(\gamma)}\right)}{d \tilde{v}^{(\gamma)}}\left\langle\tilde{v}_{, t}^{(\gamma)}\right\rangle_{+}
\end{aligned}
$$

\section{Computational Implementation}

Boxes 1 and 2 summarize the microchronological- and macrochronological-reduced order multiscopic problems, respectively. Given the coupling terms, the micro- and macrochronological problems are evaluated using the nonlinear finite element method. A commercial finite element software 


\section{Microchronological Problem:}

Given: Coefficient tensors, $\overline{\mathbf{L}}^{(\Delta)}, \overline{\mathbf{P}}^{(\Delta \alpha)}, \hat{\mathbf{A}}^{(\alpha \Delta)}$ and $\hat{\mathbf{B}}^{(\alpha \Delta \gamma)}$; average body force, $\overline{\mathbf{b}}$, boundary data $\hat{\mathbf{u}}$ and $\hat{\mathbf{t}}$

Find: at a fixed macrochronological time $\breve{t} \in\left[0, t_{f}\right]$ the macroscopic displacement field, $\overline{\mathbf{u}}$, which satisfies $\left(\tau \in\left[0, \tau_{0}\right]\right)$ :

$$
\begin{gathered}
\text { Equilibrium : } \nabla_{\mathbf{x}} \cdot \overline{\boldsymbol{\sigma}}(\mathbf{x}, \breve{t}, \tau)+\overline{\mathbf{b}}(\mathbf{x})=\mathbf{0} ; \quad \mathbf{x} \in \Omega \\
\text { Constitutive Equation: } \quad \overline{\boldsymbol{\sigma}}=\sum_{\Delta=1}^{n}\left[1-\omega^{(\Delta)}\right]\left[\overline{\mathbf{L}}^{(\Delta)}: \overline{\boldsymbol{\epsilon}}+\sum_{\alpha=1}^{n} \overline{\mathbf{P}}^{(\Delta \alpha)}: \boldsymbol{\mu}^{(\alpha)}\right] \\
\sum_{\Delta=1}^{n}\left\{\left[1-\omega^{(\Delta)}\right]\left[\hat{\mathbf{A}}^{(\alpha \Delta)}: \overline{\boldsymbol{\epsilon}}+\sum_{\gamma=1}^{n} \hat{\mathbf{B}}^{(\alpha \Delta \gamma)}: \boldsymbol{\mu}^{(\gamma)}\right]\right\}=\mathbf{0} \quad \forall \alpha=1,2, \ldots, n \\
\text { Damage Evolution: } \quad \omega_{, \tau}^{(\gamma)}=f^{0}=g^{p} \frac{d \Phi\left(v^{(\gamma)}\right)}{d v^{(\gamma)}\left\langle v_{, \tau}^{(\gamma)}\right\rangle_{+}} \\
\text {Boundary conditions: } \overline{\mathbf{u}}=\hat{\mathbf{u}}(\mathbf{x}, \breve{t}, \tau) ; \quad \mathbf{x} \in \Gamma_{u} \\
\overline{\boldsymbol{\sigma}} \cdot \mathbf{n}=\hat{\mathbf{t}}(\mathbf{x}, \breve{t}, \tau) ; \quad \mathbf{x} \in \Gamma_{t}
\end{gathered}
$$

Box 1: The microchronological system of equations for evaluation of a single load cycle.

(Abaqus) along with the user material subroutine utility (UMAT) is employed to solve these problems. In this section, we focus on the implementation details of the coupling between the microand macrochronological problems. The proposed solution strategy is implemented with an adaptive macrochronological time stepping methodology.

The overall solution strategy for the evaluation of the coupled multiscale system is illustrated in Fig. 3. Consider a discretization of the macrochronological time domain, $\left\{t_{0}=0, \ldots, t_{i-1}, t_{i}, t_{i+1}, \ldots, t_{k}=t_{f}\right\}$ in which $t_{i}$ denotes the $i^{\text {th }}$ macrochronological time step:

$$
t_{i}=\sum_{j=1}^{i} \Delta t_{j} \leq t_{f}
$$

where, $\Delta t_{i}=t_{i}-t_{i-1}$ and $t_{0}=0$. A driver program (implemented in the Python programming language for compatibility with Abaqus) controls the execution of the solution procedure. At each macrochronological time step, the time step size as well as the almost periodic damage field are estimated and passed to the macrochronological - multiscopic problem, which is evaluated for macrochronological response fields. The macrochronological response fields provide the initial state of the microchronological multiscopic problem at fixed time $t_{i}$ due to the particular choice of the temporal homogenization operator. The point-wise value of the almost periodic damage field, $\omega_{\mathrm{ap}}^{(\gamma)}$, at the current time step is computed and passed to the driver routine for computation of the next time step size.

\subsection{Adaptive Macrochronological Time Stepping}

The proposed solution algorithm outlined in Fig. 3 requires the resolution of $k$ cycles throughout the loading history, which are evaluated by the microchronological problem (Box 1). The accuracy and efficiency of the proposed approach is based on the appropriate selection of the macrochronological time steps, as well as the accurate approximation of the evolution of the almost periodic component of 


\section{Macrochronological Problem:}

Given: coefficient tensors, $\overline{\mathbf{L}}^{(\Delta)}, \overline{\mathbf{P}}^{(\Delta \alpha)}, \hat{\mathbf{A}}^{(\alpha \Delta)}$ and $\hat{\mathbf{B}}^{(\alpha \Delta \gamma)}$; average body force, $\overline{\mathbf{b}}$, boundary data, $\hat{\mathbf{u}}^{0}$ and $\hat{\mathbf{t}}^{0}$; the almost periodic damage function, $\omega_{\text {ap }}$.

Find: the displacement field, $\tilde{\overline{\mathbf{u}}}$, which satisfies $\left(t \in\left[0, t_{f}\right]\right)$ :

$$
\text { Equilibrium : } \nabla_{\mathbf{x}} \cdot \tilde{\tilde{\sigma}}(\mathbf{x}, t)+\overline{\mathbf{b}}(\mathbf{x})=\mathbf{0} ; \quad \mathbf{x} \in \Omega
$$

$$
\begin{aligned}
& \text { Constitutive Equation: } \tilde{\tilde{\boldsymbol{\sigma}}}=\sum_{\Delta=1}^{n}\left[1-\tilde{\boldsymbol{\omega}}^{(\Delta)}\right]\left[\overline{\mathbf{L}}^{(\Delta)}: \tilde{\overline{\boldsymbol{\epsilon}}}+\sum_{\alpha=1}^{n} \overline{\mathbf{P}}^{(\Delta \alpha)}: \tilde{\boldsymbol{\mu}}^{(\alpha)}\right] \\
& \sum_{\Delta=1}^{n}\left\{\left[1-\tilde{\boldsymbol{\omega}}^{(\Delta)}\right]\left[\hat{\mathbf{A}}^{(\alpha \Delta)}: \overline{\boldsymbol{\epsilon}}+\sum_{\gamma=1}^{n} \hat{\mathbf{B}}^{(\alpha \Delta \gamma)}: \tilde{\boldsymbol{\mu}}^{(\gamma)}\right]\right\}=\mathbf{0} \quad \forall \alpha=1,2, \ldots, n
\end{aligned}
$$

Damage Evolution: $\dot{\tilde{\omega}}(\mathbf{x}, t)=f^{1}+\omega_{\mathrm{ap}}=g^{p} \frac{d \Phi\left(\tilde{\mathrm{v}}^{(\gamma)}\right)}{d \tilde{\mathrm{v}}^{(\gamma)}}\left\langle\tilde{\mathrm{v}}_{, t}^{(\gamma)}\right\rangle_{+}+\omega_{\mathrm{ap}}(\mathbf{x}, t) ; \quad \mathbf{y} \in \theta$

$$
\text { Boundary conditions: } \tilde{\tilde{\mathbf{u}}}=\hat{\mathbf{u}}^{0}(\mathbf{x}, t) ; \quad \mathbf{x} \in \Gamma_{u}
$$

$$
\tilde{\tilde{\boldsymbol{\sigma}}}(\mathbf{x}, t) \cdot \mathbf{n}=\hat{\mathbf{t}}^{0}(\mathbf{x}, t) ; \quad \mathbf{x} \in \Gamma_{t}
$$

Box 2: The macrochronological system of equations for evaluation of a macroscale time step.

the damage field. In this study, the almost periodic damage fields are approximated based on a modified quadratic multistep method [35], whereas the macrochronological time step size is chosen adaptively based on a maximum damage accumulation criterion. It is assumed that damage accumulation is primarily due to cyclic loading and slow loading component remain smooth during the loading history.

Let $\mathbf{D}^{\prime}(t)$ be a matrix of almost periodic damage rate fields:

$$
\mathbf{D}^{\prime}(t)=\left[\begin{array}{ccc}
\omega_{\mathrm{ap}}^{(1)}\left(\mathbf{x}_{1}, t\right) & \cdots & \omega_{\mathrm{ap}}^{(n)}\left(\mathbf{x}_{1}, t\right) \\
\vdots & \ddots & \vdots \\
\omega_{\mathrm{ap}}^{(1)}\left(\mathbf{x}_{n_{g}}, t\right) & \cdots & \omega_{\mathrm{ap}}^{(n)}\left(\mathbf{x}_{n_{g}}, t\right)
\end{array}\right]
$$

in which, $n_{g}$ denotes the total number of integration points within the macroscopic domain; $\mathbf{x}_{g}$ denotes the value of the function at integration point $g$. Considering a smooth damage growth between macrochronological steps $i-1$ and $i+1$, the evolution of $\mathbf{D}^{\prime}(t)$ is approximated by a linear function around $t_{i}$ :

$$
\mathbf{D}^{\prime}\left(t_{i}+\Delta t\right) \approx \mathbf{P}_{i}(\Delta t) \equiv \mathbf{b}_{i}+\mathbf{c}_{i} \Delta t ; \quad \Delta t \in\left[-\Delta t_{i}, \Delta t_{i+1}\right]
$$

The coefficients of the linear approximations are obtained by imposing the following conditions on $\mathbf{P}_{i}$ :

$$
\begin{gathered}
\mathbf{P}_{i}(0)=\mathbf{D}^{\prime}\left(t_{i}\right) \\
\mathbf{P}_{i}\left(-\Delta t_{i}\right)=\mathbf{D}^{\prime}\left(t_{i-1}\right)
\end{gathered}
$$

Substituting Eqs. 41, and 42 into Eq. 40, the rate of the almost periodic damage fields is obtained as:

$$
\mathbf{D}^{\prime}\left(t_{i+1}\right) \approx \mathbf{P}\left(\Delta t_{i+1}\right)=\mathbf{D}^{\prime}\left(t_{i}\right)+\frac{\Delta t_{i+1}}{\Delta t_{i}}\left(\mathbf{D}^{\prime}\left(t_{i}\right)-\mathbf{D}^{\prime}\left(t_{i}-1\right)\right)
$$

Let $\Delta \mathbf{D}_{i+1}$ define the matrix of cyclic loading induced damage accumulated between time $t_{i}$ and $t_{i+1}$. Employing the linear approximation provided by Eq. 40 yields:

$$
\Delta \mathbf{D}_{i+1} \approx \int_{0}^{\Delta t_{i+1}} \mathbf{P}_{i}(\Delta t) d \Delta t=\Delta t_{i+1} \mathbf{D}^{\prime}\left(t_{i}\right)+\frac{\Delta t_{i+1}^{2}}{2 \Delta t_{i}}\left(\mathbf{D}^{\prime}\left(t_{i}\right)-\mathbf{D}^{\prime}\left(t_{i}-1\right)\right)
$$




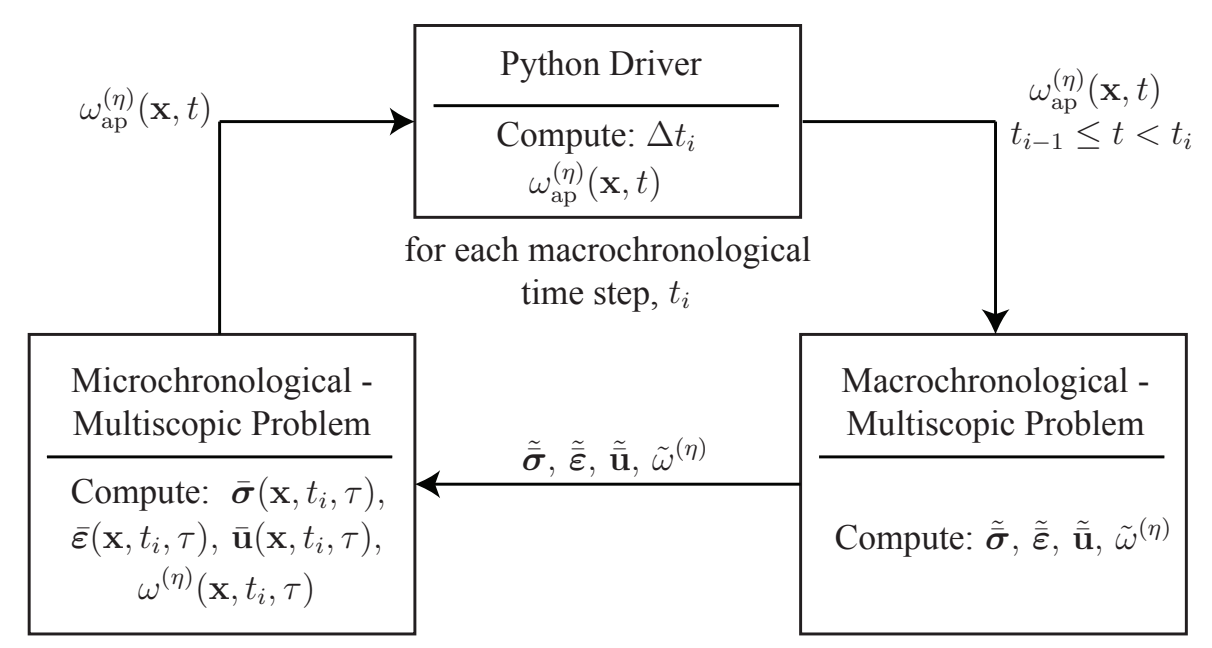

Figure 3: Implementation strategy of the coupled micro- and macro-chronological problems.

The macrochronological step size $\left(\Delta t_{i+1}\right)$ is chosen such that the maximum damage accumulation within a step does not exceed a threshold value:

$$
\left\|\Delta \mathbf{D}_{i+1}\right\|_{\max } \leq \Delta D_{\max }
$$

where, $\Delta D_{\max }$ is the damage accumulation threshold, and $\|\cdot\|_{\max }$ denotes the matrix max norm.

The loss of load carrying capacity during a microchronological problem following a long macrochronological step indicates a possible underestimation of damage accumulation during the macrochronological time step. This is due to the deviation of the damage accumulation from the piecewise quadratic approximation within the time step. When failure is observed, the previous macrochronological step size is shortened, and the previous macrochronological time step is repeated. The time step shortening is repeated until desired accuracy of the time-to-failure is achieved. The overall algorithm is presented below:

1. Initialize algorithm: $i=0$.

2. Evaluate macrochronological step (Box 2).

3. Evaluate microchronological problem at $t=0(\operatorname{Box} 1)$.

4. Set $i=1 ; \Delta t_{1}=\eta \tau_{0}$, where $\eta \tau_{0}$ is the duration of one load cycle; $\mathbf{D}^{\prime}\left(t_{1}\right)=\mathbf{D}^{\prime}(0)$.

5. Evaluate macrochronological step.

6. Evaluate microchronological problem at $t=t_{1}$.

7. Set $i=2$

8. While $i \leq k$ :

(a) Calculate $\Delta t_{i}$ using Eq. 45.

(b) Calculate $\mathbf{D}^{\prime}\left(t_{i}\right)$ using Eq. 43.

(c) Evaluate macrochronological step.

(d) Evaluate microchronological problem at $t=t_{i}$.

(e) If $\left\{\right.$ failure event $\left.\& \Delta t_{i} / t_{i} \geq t o l\right\}$ :

i. $\Delta t_{i} \leftarrow \max \left(\eta \tau_{0}, c \Delta t_{i}\right)$ and go to step (b). 
(f) Else if $\left\{\right.$ failure event $\left.\& \Delta t_{i} / t_{i}<t o l\right\}$ :

i. Structural failure: Stop algorithm.

(g) $i \leftarrow i+1$

9. End.

The proposed algorithm is initiated by evaluating two macrochronological time steps and two microchronological problems that corresponds to the first two load cycles, noting that a single load period is denoted by $\eta \tau_{0}$. The almost periodic field is taken to vary linearly between the first and second load cycles, and quadratically between the remaining macrochronological time steps. $0<c<1$ is the step cutback factor when a failure event is detected. The failure event is defined as a loss of load carrying capacity of the structure, detected as lack of convergence that occurs during the evaluation of the microchronological problem. The macrochronological time step size cannot be smaller than a single load period $\left(=\eta \tau_{0}\right)$. Structural failure is taken to occur when the failure event is detected, and the ratio of the current macrochronological time step size and the current macrochronological time is less than a specified tolerance $(t o l)$ value. The cutback iterations when a failure event is detected are controlled by the Python driver program, which employs the restart capability of Abaqus to iterate the evaluation of macro- and micro-chronological problems until convergence.

\section{Model Verification}

The proposed adaptive macrochronological time stepping strategy is verified by comparing the performance with direct cycle-by-cycle simulations. In the direct cycle-by-cycle analysis, each load cycle throughout the loading is resolved, without resorting to the multiple temporal scale strategy. The simulations were conducted on a unidirectionally fiber-reinforced matrix unit cell. The fiber is taken to remain elastic throughout the loading period, whereas the damage accumulates within the matrix as a function of loading cycles. The failure response in the matrix phase is approximated using a 4-partition reduced-order model. A uniform tensile strain is applied transverse to the reinforcement direction. The strain amplitude is varied between zero and the maximum strain throughout the loading history.

Figure 4 illustrates the variation of the damage variables within the unit cell as a function of applied loading cycles. Only two of the four matrix partitions show appreciable damage. The proposed model simulations are conducted by setting $\Delta D_{\max }=0.5 \%, 1 \%$, and $2 \%$. A cutback factor of 0.5 is employed $(c=0.5)$. The proposed adaptive time stepping strategy required 109, 68, and 43 resolved microchronological load cycles for $\Delta D_{\max }=0.5 \%, 1 \%$, and $2 \%$, respectively, compared to 900 cycles resolved in the direct cycle-by-cycle approach. The proposed model captures the failure response with reasonable accuracy, with excellent accuracy observed for $\Delta D_{\max }=0.5 \%$.

The efficiency of the proposed approach is further illustrated by conducting simulations when the unit cell is subjected to a slightly smaller loading amplitude. In this analysis, the cycle-to-failure of the first partition is 677 (in contrast to 377 of the previous simulations). The simulations are conducted for 1800 cycles. Figure 5 illustrates the variation of damage variables within the unit cell as a function of applied loading cycles as computed by the reference simulations and the multiple spatiotemporal model with adaptive time stepping methodology. The total resolved cycles of the proposed adaptive time stepping strategy remain largely the same $\left(\Delta D_{\max }=0.5 \%, 1 \%\right.$, and $2 \%$ are 118,72 and 47 respectively), pointing to significant computational advantage in high cycle failure conditions. 


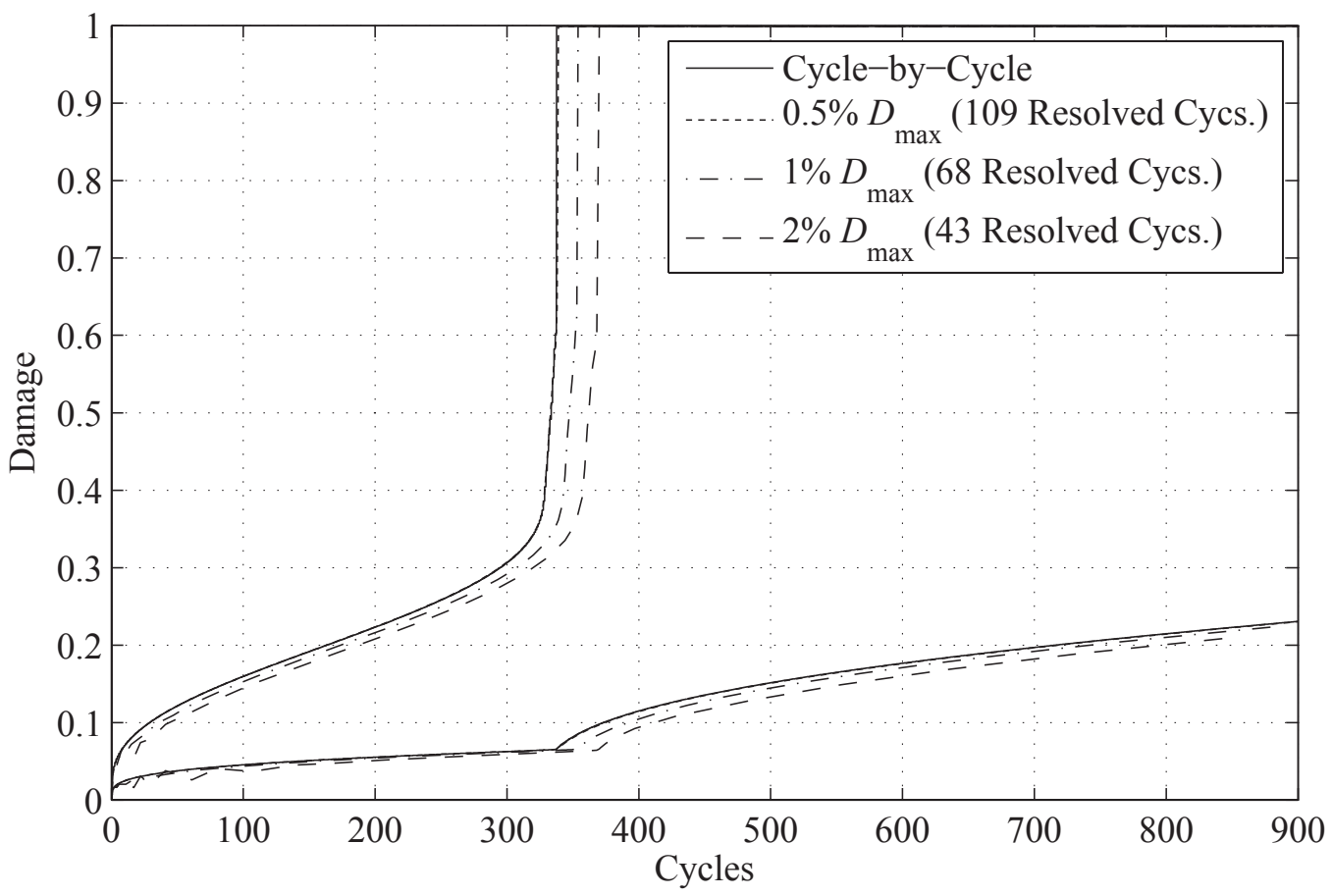

Figure 4: Comparison of the cyclic damage accumulation computed using the direct cycle-bycycle approach and proposed multiscale model with adaptive time stepping.

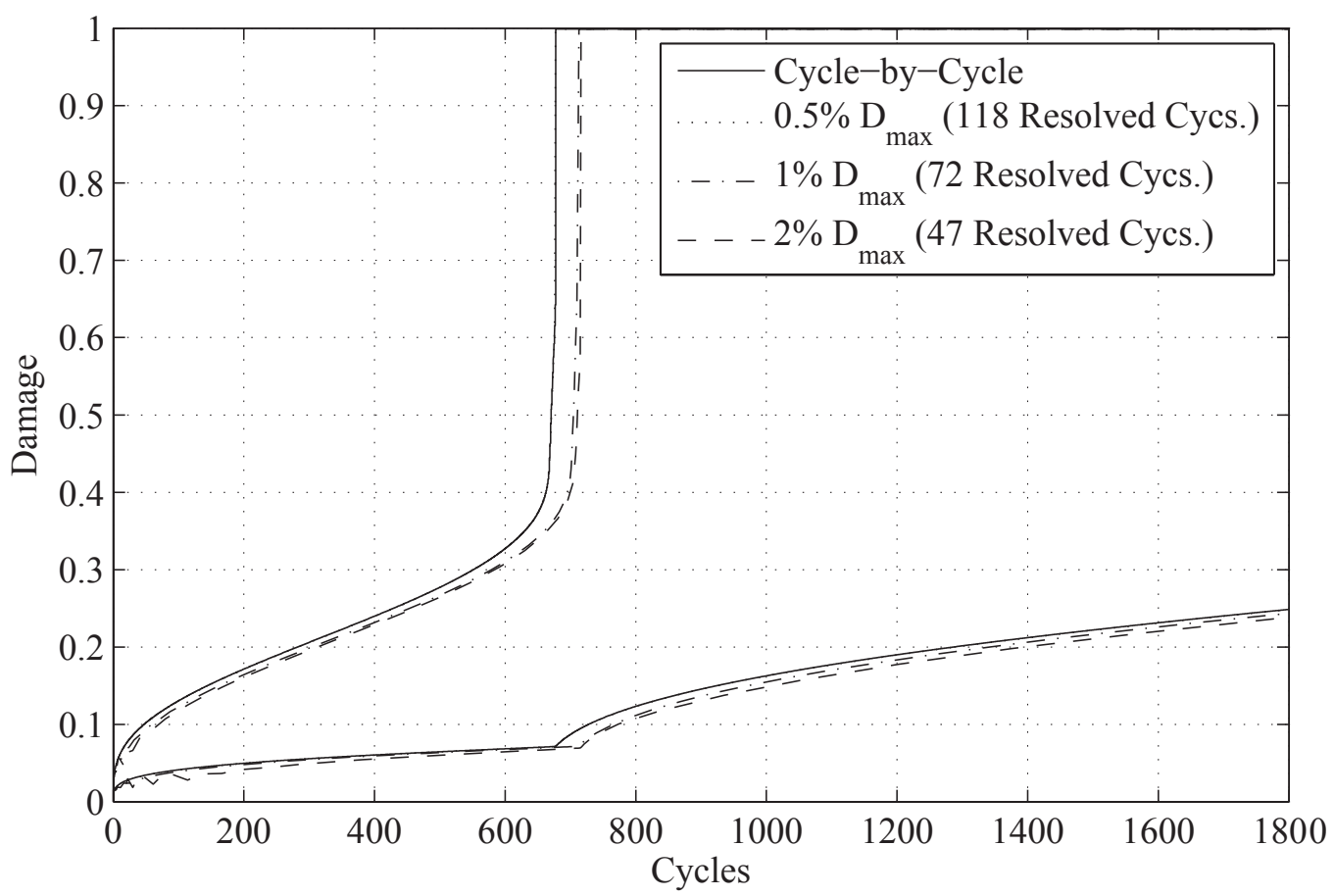

Figure 5: Comparison of the cyclic damage accumulation computed using the direct cycle-bycycle approach and proposed multiscale model with adaptive time stepping with smaller loading amplitude. 
Table 1: IM7/977-3 specimen dimensions

\begin{tabular}{ccccc}
\hline $\begin{array}{c}\text { Fiber } \\
\text { Orientation }\end{array}$ & $\begin{array}{c}\text { Number } \\
\text { Of Plies }\end{array}$ & $\begin{array}{c}\text { Length } \\
{[\mathrm{mm}]}\end{array}$ & $\begin{array}{c}\text { Width } \\
{[\mathrm{mm}]}\end{array}$ & $\begin{array}{c}\text { Thickness } \\
{[\mathrm{mm}]}\end{array}$ \\
\hline $0^{\circ}$ & 8 & 250 & 13 & 1 \\
$90^{\circ}$ & 16 & 177 & 25 & 2 \\
Quasi-Iso. & 16 & 250 & 25 & 2 \\
\hline
\end{tabular}

\section{Cyclic Response of IM7/977-3 Composites}

The capabilities of the proposed multiple spatio-temporal methodology is assessed through the investigation of graphite fiber-reinforced epoxy composites (i.e., IM7/977-3) subjected to cyclic loading. This section presents the experiments conducted to study the cyclic response of the composite; calibration of the model parameters based on monotonic and cyclic experiments, and; validation of model predictions based on acoustic emission testing.

\subsection{Experiments}

A suite of experiments was conducted to calibrate the material parameters and assess the validity of the proposed multiscale model. Composite specimens with three separate layups of unidirectional laminae were tested under uniaxial monotonic and cyclic loading conditions: (a) Zero degree specimens consist of eight unidirectional plies with fibers oriented parallel to the loading direction; (b) Ninety degree specimens consist of sixteen unidirectional plies with the fibers oriented perpendicular to the loading direction; and (c) Quasi-isotropic specimens with the layup of $[+45,0,-45,90]_{2 s}$. Specimen configurations are summarized in Table 2. The mean fiber volume fraction of the specimens is $65.6 \%$, which was determined based on acid digestion testing. The results of experiments conducted on zero and ninety degree specimens are employed in the calibration of the parameters of the proposed multiscale model, whereas the quasi-isotropic specimens are employed in the validation analyses.

Acoustic emission (AE) was used to detect failure events within the quasi-isotropic layups. In-situ AE activity was recorded on a Micro-II Digital AE System produced by Physical Acoustics Corporation. In the AE technique, the stress waves produced by the sudden release of strain energy during localized failure events are identified and recorded as hits. Appropriate signal conditioning parameters are identified based on an AE calibration study prior to testing. A threshold wave amplitude of $48 \mathrm{~dB}$ enables the separation of all valid failure events from ambient noise.

The first set of experiments conducted is on unidirectional unnotched tension specimens tested under monotonic displacement control on an MTS universal testing machine according to ASTM Standard D3039 [36]. A grip pressure of 500 psi was applied to prevent slipping without crushing the composite. One axial and one transverse strain gage were mounted on each specimen to determine Poisson's ratio. A one-inch extensometer was used to accurately measure the axial stiffness. All monotonic tests were conducted at a constant displacement rate of $1.27 \mathrm{~mm} / \mathrm{min}$. Thirteen zero degree specimens, seventeen ninety degree specimens, and seven quasi-isotropic specimens were subjected to uniaxial tension up to failure to ensure repeatibility. A moderate degree of modulus and strength scatter is observed in the experiments. Figure 6 illustrates the failure patterns, which show destructive fiber failure in the zero degree specimens, matrix-dominated failure in the ninety degree specimens, and combined matrix and fiber failure in the quasi-isotropic specimens. All non-zero plies in the quasiisotropic specimens showed matrix-dominated failure, while the zero degree plies showed fiber failure. 


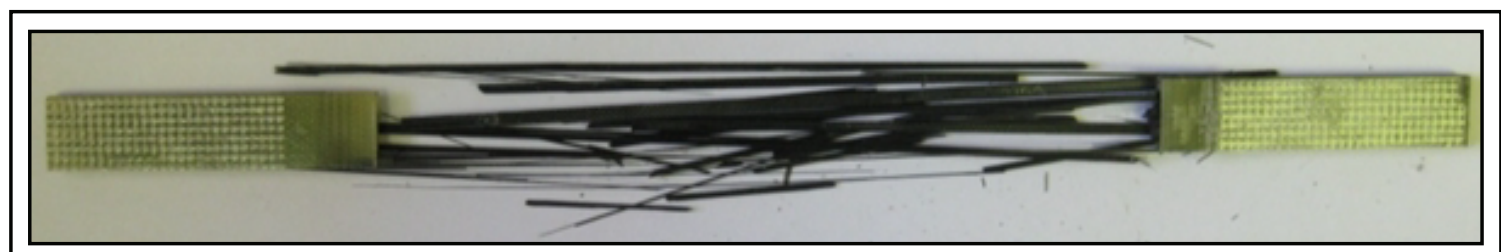

(a)

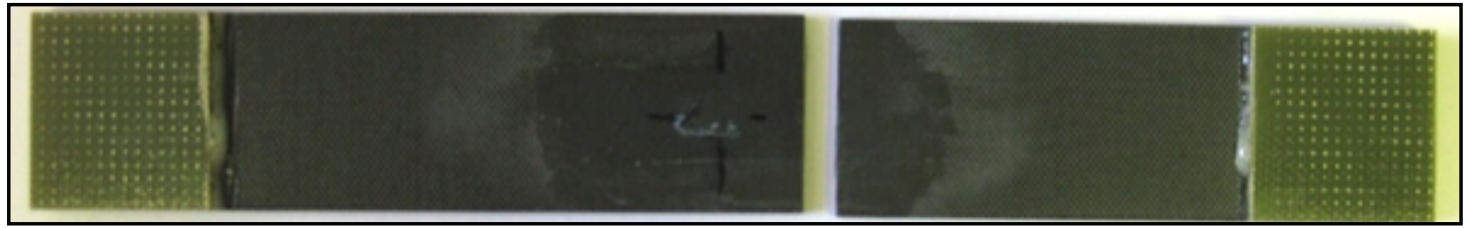

(b)

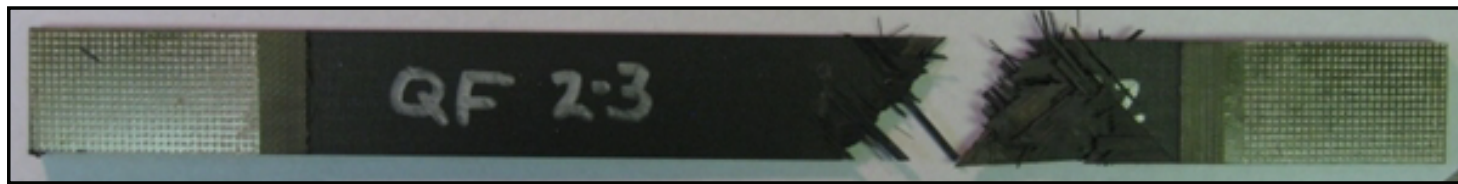

(c)

Figure 6: Failure profiles when subjected to monotonic loading. (a) Zero-degree specimens; (b) ninety degree specimens; (c) quasi-isotropic specimens.

Table 2: Calibrated elastic parameters of the composite constituents; observed and simulated elastic parameters of the overall composite.

\begin{tabular}{ccc}
\hline Layup & $\begin{array}{c}\text { Young's Modulus, } E \\
{[\mathrm{GPa}]}\end{array}$ & $\begin{array}{c}\text { Failure Strength, } \sigma_{\mathrm{f}} \\
{[\mathrm{MPa}]}\end{array}$ \\
\hline $0^{\circ}$ & $158(13)^{1}$ & $2,841(296)$ \\
$90^{\circ}$ & $8.644(0.712)$ & $63(14)$ \\
Quasi-isotropic & $60.7(2.2)$ & $872(30)$ \\
\hline
\end{tabular}

${ }^{1}$ standard deviation in parantheses.

The elastic and strength properties observed in the experiments are summarized in Table 2. A Poisson's ratio of 0.316 with a standard deviation of 0.039 was observed by placing a strain gage perpendicular to the loading on the $0^{\circ}$ specimens. The shear modulus, $G_{12}$, was determined to be $4.66 \mathrm{MPa}$ with a standard deviation of 0.61 using additional tension experiments conducted on composite specimens with a $+/-45^{\circ}$ layup according to the procedure described in ASTM D3518 [37].

The next set of experiments consisted of constant amplitude load-controlled cyclic tests that were conducted according to ASTM D3479 [38]. Cyclic testing was performed with a constant maximum stress amplitude, an R-ratio of 0.1 , and a loading frequency of $5 \mathrm{~Hz}$. The maximum applied stress amplitude for the $90^{\circ}$ specimens was varied between $45 \%$ and $55 \%$ of the average monotonic ultimate stress of the layup configuration. The $90^{\circ}$ specimens failed by matrix cracking across the width of the specimen. The quasi-isotropic layup was tested with a maximum applied stress amplitude of $17 \%$ of the ultimate stress of the corresponding layup. 


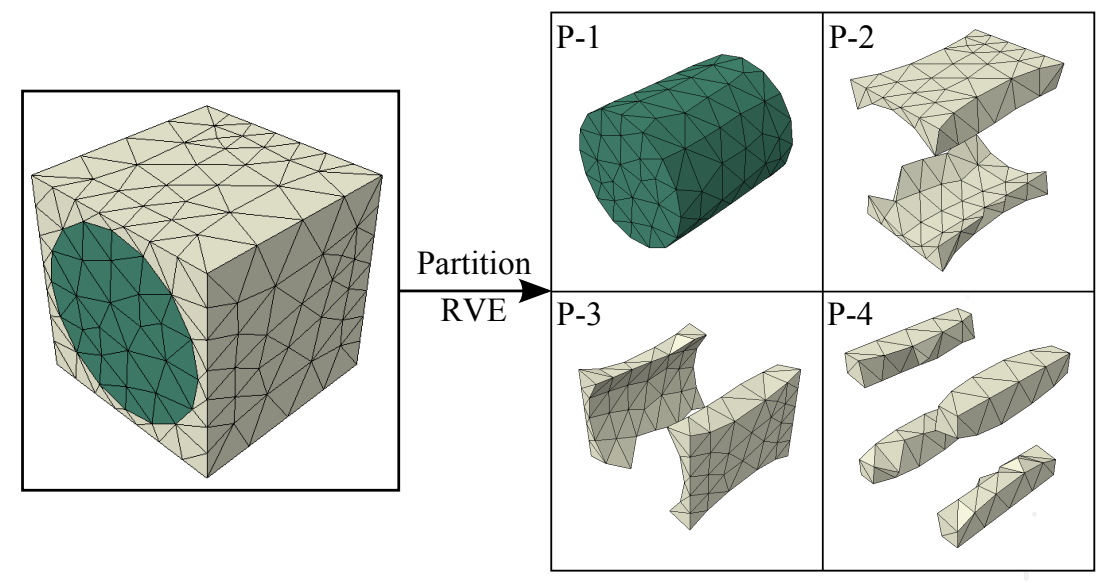

Figure 7: RVE and partition structure for IM7/977-3.

Table 3: Elastic parameter optimization

\begin{tabular}{ccccccc}
\hline$E^{\mathrm{m}}[\mathrm{GPa}]$ & $E_{1}^{\mathrm{f}}[\mathrm{GPa}]$ & $E_{2}^{\mathrm{f}}[\mathrm{GPa}]$ & $G_{12}^{\mathrm{f}}[\mathrm{GPa}]$ & $v^{\mathrm{m}}$ & $v_{12}^{\mathrm{f}}$ & $v_{23}^{\mathrm{f}}$ \\
\hline 3.55 & 263.00 & 13.00 & 27.50 & 0.35 & 0.32 & 0.20 \\
\hline \hline & $E_{1}^{\mathrm{c}}[\mathrm{GPa}]$ & $E_{2}^{\mathrm{c}}[\mathrm{GPa}]$ & $G_{12}^{\mathrm{c}}[\mathrm{GPa}]$ & $v_{12}^{\mathrm{c}}$ & & \\
\hline Experiment & 158.00 & 8.64 & 4.66 & 0.316 & & \\
Model & 158.00 & 8.64 & 4.66 & 0.33 & & \\
\hline
\end{tabular}

Superscript $c$ indicates a composite material property.

\subsection{Model Calibration}

The domain of the microscopic problem (i.e., RVE) is a unidirectionally fiber reinforced matrix as illustrated in Fig. 7. The diameter of the fiber is set to ensure that the volume fraction in the RVE equals the experimentally measured volume fraction of $65.6 \%$. We employ a 4-partition reduced order model to evaluate the failure response within the composite constituents. The matrix is represented using three partitions, whereas the fiber response is idealized using a single partition. The domains of each partition within the RVE are illustrated in Fig. 7.

The elastic response of the 977-3 resin is taken to be isotropic, with the Young's modulus and the Poisson's ratio of the material denoted by $E^{\mathrm{m}}$ and $v^{\mathrm{m}}$, respectively. The IM7 fiber is taken to be transversely isotropic with five elastic parameters: $E_{1}^{\mathrm{f}}, E_{2}^{\mathrm{f}}, G_{12}^{\mathrm{f}}, v_{12}^{\mathrm{f}}$, and $v_{23}^{\mathrm{f}}$, where the 1-direction is along the fiber length. The Poisson's ratios were obtained from the literature $\left(v_{12}^{\mathrm{f}}\right.$ and $v_{23}^{\mathrm{f}}$ from [39] and $v^{\mathrm{m}}$ from [2]). The remaining elastic parameters (i.e., $E_{1}^{\mathrm{f}}, E_{2}^{\mathrm{f}}, G_{12}^{\mathrm{f}}$, and $E^{\mathrm{m}}$ ) were calibrated against the linear regions of the stress-strain curves recorded in the monotonic experiments. The calibrated elastic parameters of the composite constituents and the experimentally observed and simulated elastic parameters of the overall composite are summarized in Table 3.

The damage model employed in this study includes seven parameters. Four of the seven parameters (i.e., $\alpha^{\mathrm{m}}, \beta^{\mathrm{m}}, \alpha^{\mathrm{f}}$, and $\beta^{\mathrm{f}}$ ) determine the evolution of damage when subjected to monotonic loading conditions, whereas the remaining three parameters (i.e., $c_{0}^{\mathrm{m}}, c_{1}^{\mathrm{m}}$ and $c_{2}^{\mathrm{m}}$ ) determine the sensitivity of damage evolution to cyclic loading. Experiments conducted under cyclic tensile conditions indicate that failure initiates within the matrix. Fibers are taken to be insensitive to cyclic failure at the loading 


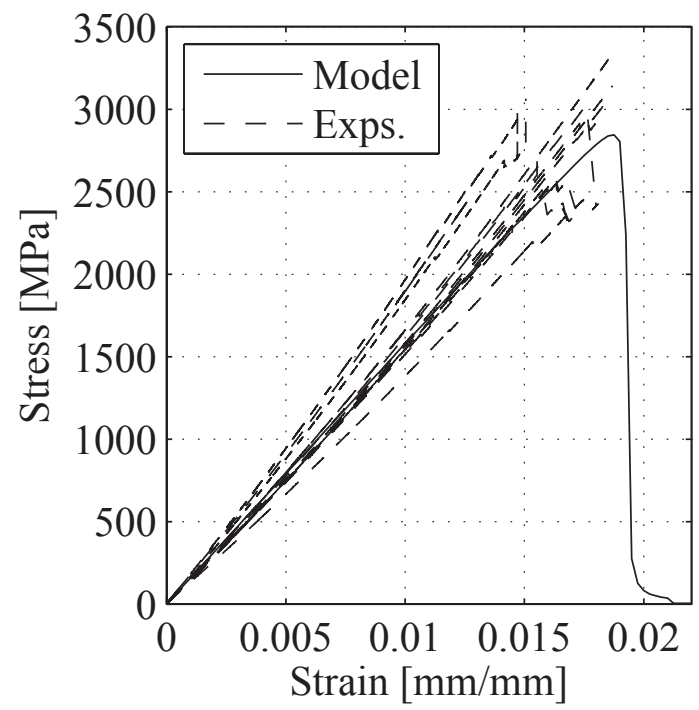

(a)

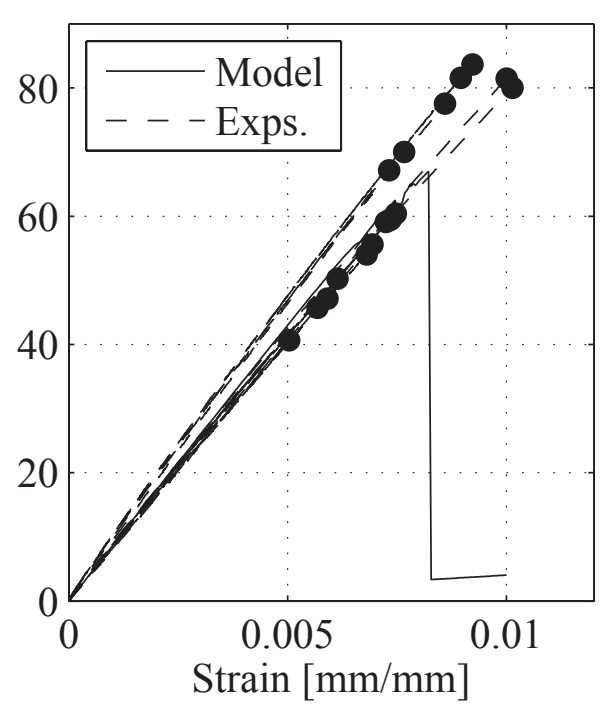

(b)

Figure 8: The tension experiments are compared to the calibrated model response.

amplitudes considered in this work. The superscripts $\mathrm{m}$ and $\mathrm{f}$ denote matrix and fiber phases respectively. The matrix parameters are employed in modeling failure in the three matrix partitions, whereas the fiber parameters are employed in modeling the fiber partition.

$\alpha^{\mathrm{m}}$ and $\alpha^{\mathrm{f}}$ are regularization parameters that control the abruptness of the ultimate failure within the matrix and fiber phases, respectively. The values of $\alpha^{\mathrm{m}}$ and $\alpha^{\mathrm{f}}$ are chosen to avoid numerical difficulties associated with sudden failure events, while accurately capturing the characteristics of the stress-strain response. $\beta^{\mathrm{m}}$ and $\beta^{\mathrm{f}}$ are material parameters that control the ultimate strength of the matrix and fiber, respectively. The experimentally observed stress-strain response of the zero and ninety degree specimens are employed in the calibration process. The failure in the zero degree specimens is dominated by fiber failure, whereas matrix cracking dominates failure in the ninety degree specimens subjected to monotonic loading. $\beta^{\mathrm{m}}$ and $\beta^{\mathrm{f}}$ are identified by minimizing the discrepancy between the experimental and simulated stress-strain curves. The calibrated model parameters are $\alpha^{\mathrm{m}}=0.05, \beta^{\mathrm{m}}=$ $32.0, \alpha^{\mathrm{f}}=0.05$, and $\beta^{\mathrm{f}}=340.0$. Figure 8 illustrates the experimentally observed and simulated stressstrain curves based on calibrated material parameters for zero degree and ninety-degree specimens. The mean ultimate strength and strain-at-failure for zero degree specimens based on experiments are $2841 \mathrm{MPa}$ and 0.0180 , respectively. The mean ultimate strength and strain-at-failure for ninety degree specimens based on experiments are $63 \mathrm{MPa}$ and 0.00728 , respectively. The calibrated model yields $2846 \mathrm{MPa}$ and 0.0186 for zero degree loadings and $67 \mathrm{MPa}$ and 0.00822 for ninety degree loadings, which are in close agreement with the experiments.

The cyclic failure of the matrix is characterized by the three remaining parameters: $c_{0}^{\mathrm{m}}, c_{1}^{\mathrm{m}}$ and $c_{2}^{\mathrm{m}}$. The calibration of the cyclic loading sensitivity parameters is conducted by employing the stress-life curves obtained from experiments in which ninety degree specimens are subjected to cyclic loading in the tensile direction. In the experiments, the maximum amplitude of the cyclic loading was varied between $360 \mathrm{MPa}$ and $520 \mathrm{MPa}$, while keeping the R-ratio constant (=0.1). The three parameters are calibrated by minimizing the discrepancy between the experimental and simulated cycles to failure under three different loading amplitudes. A least squares nonlinear optimization algorithm is employed in the identification of the optimal parameters. The calibrated values of $c_{0}, c_{1}$, and $c_{2}$ are 8.243, -13.36, and 7.891, respectively. Figure 9 illustrates the experimentally observed and simulated life curves. The 


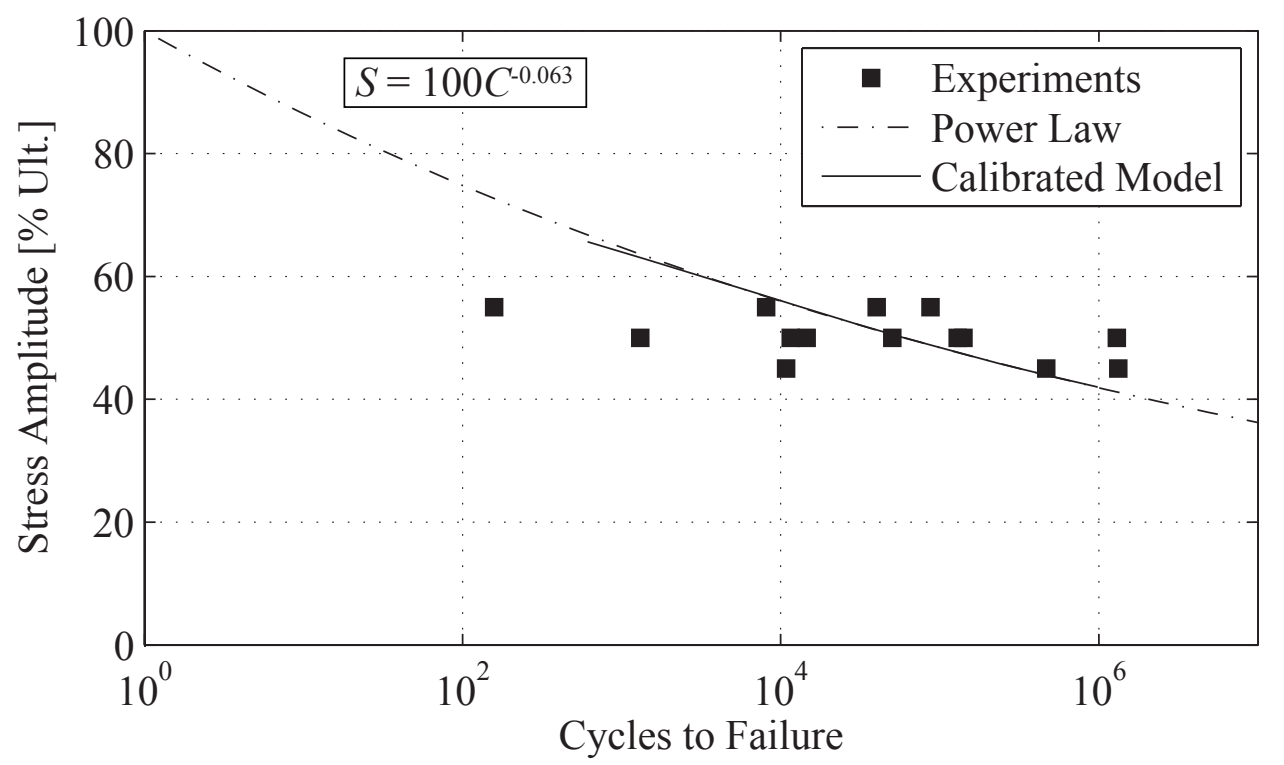

Figure 9: Experimentally observed and simulated stress-life curves of the ninety degree specimens.

calibrated model is in close agreement with the experimentally observed mean stress-life curve, which is expressed in terms of a power law fit. We note that the experiments display a substantial scatter around the power law fit for this material.

\subsection{Model Validation}

The capabilities of the proposed multiple spatio-temporal modeling approach are validated by comparing the predictions of the calibrated model with experiments conducted on quasi-isotropic specimens subjected to monotonic and cyclic loading conditions. Quarter of the specimen geometry is discretized due to symmetry with top eight plies explicitly modeled.

Figure 10 illustrates the experimental and simulated stress-strain response of the quasi-isotropic specimens subjected to monotonic tensile loading. The experimentally-observed mean strength and strain-to-failure are $872 \mathrm{MPa}$ and 0.0144 , respectively. The proposed multiscale model predictions of the strength and strain-to-failure are $872 \mathrm{MPa}$ and 0.0151 , which are in excellent agreement with each other. The simulations revealed progressive failure within the matrix of individual off-axis plies as a function of loading. The ultimate failure is due to fiber failure in the $0^{\circ}$ plies. The predicted failure pattern is in close agreement with the experimental observations. Figure 11 shows the results of the acoustic emission testing of a monotonically loaded specimen in terms of hits as a function of applied stress magnitude. The acoustic emission data shown is indicative of the response of a typical specimen. The distinct failure events predicted by our simulations are indicated as well. The orientation of the ply in which the failure event occurs is also shown in Figure 11. All of the failure events were in the matrix with the exception of the final event. Since the proposed multiscale model is calibrated to the mean response of all specimens, the provided comparison is qualitative. Despite variations, the progressive nature of the matrix damage accumulation, as well as the initiation of damage is well captured by the proposed multiscale model.

We further assessed the validity of the proposed model by comparing the model predictions to experiments on quasi-isotropic specimens subjected to cyclic loading conditions. A sinusoidal load with 


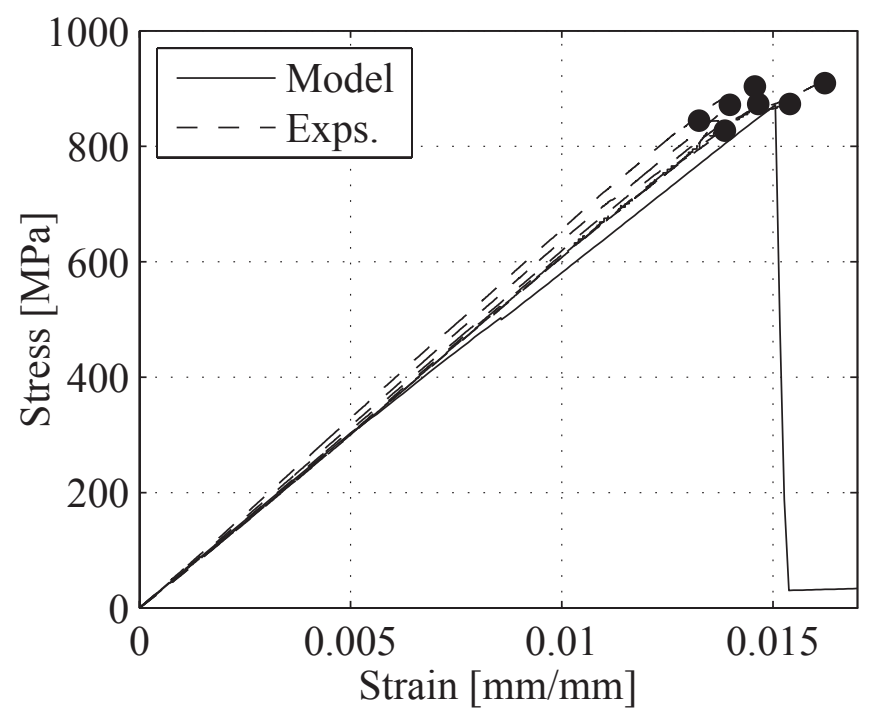

Figure 10: Comparison of experimental and predicted stress-strain curves of the quasi-isotropic specimens subjected to monotonic tensile loading.

a peak magnitude of $17 \%$ of the mean ultimate failure load of the quasi-isotropic specimens (872 MPa) and an R-ratio of 0.1 is applied. The objective of the investigation is to assess the capability of the proposed model in capturing the distinct failure events that occur within the matrix material as a function of the applied load cycles. In our simulations, the first four distinct failure events occurred at 11766, 27794, 38817 and 46694 cycles, respectively. Each of these failure events was an individual off-axis ply losing load carrying capacity due to a matrix crack extending the entire width of the specimen. The first failure event was in a ninety degree ply, the second and third events were in fourty-five degree plies, and the fourth event was in a minus fourty-five degree ply. Figure 12a shows the acoustic emission testing results in terms of total hits as a function of the number of loading cycles. The failure events predicted by the proposed multiscale model are indicated in Fig. 12a. Figure 12b plots the numerical derivative of total hits with respect to the total number of accumulated load cycles. The figures illustrate that the time-to-failure for major recorded failure events coincide with those predicted by the simulations. Despite close correlation with the acoustic emission testing, the predictive capability of the proposed model is qualitative. Additional experimental investigations that quantitatively link the failure events that occur during the cyclic loading are needed to fully assess the validity of the proposed approach.

\section{Conclusions}

A multiscale computational framework for modeling and prediction of failure in composite materials subjected to cyclic loading conditions is proposed. The reduced-order multiple spatial scale approach coupled with multiple temporal scale algorithm provides the level of computational efficiency without significant loss of accuracy, which is critical to the prediction of life and failure in large-scale composite structures. The experimentally calibrated model has the capability to predict the experimentally-observed early life failure events in carbon-fiber reinforced polymer matrix composite specimens. A number of challenges remain to be investigated. First, while the proposed multiscale approach is microscopically nonlocal, the local character of the damage evolution at the macroscale leads to spurious mesh dependency. Due to the significant associated computational costs, straightforward 


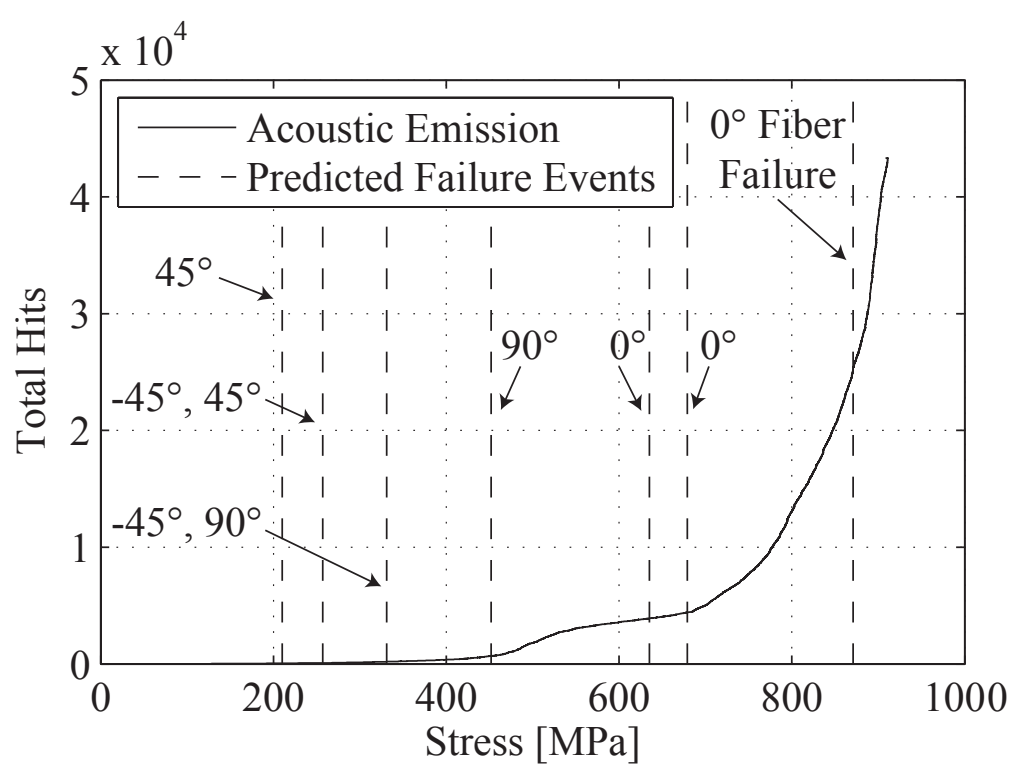

Figure 11: Predicted failure events compared to acoustic emission data of a quasi-isotropic specimen subjected to monotonic tensile loading. The ply in which the failure occurs is indicated. All predicted failure events are in the matrix unless indicated otherwise.
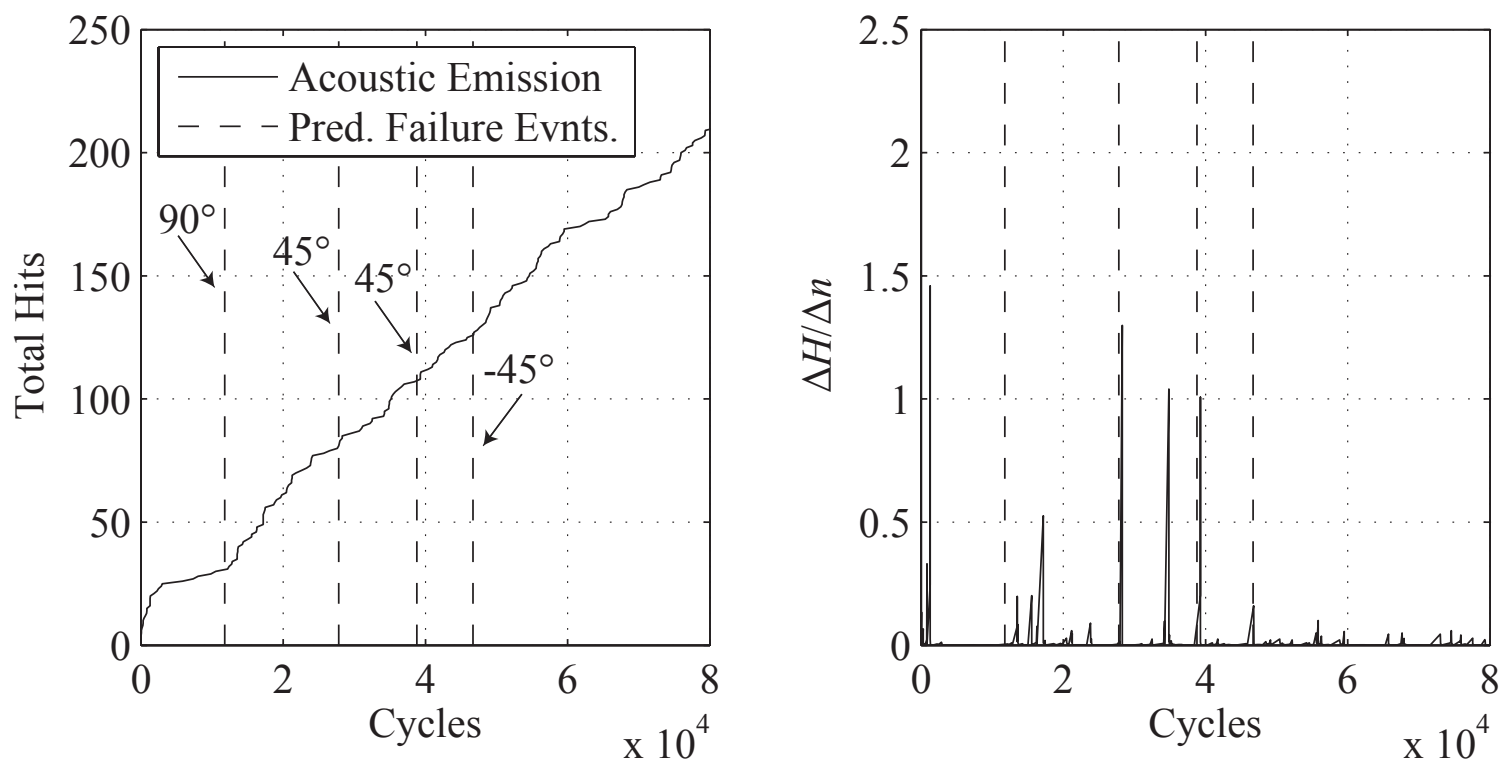

Figure 12: Predicted failure events compared to acoustic emission data of a quasi-isotropic specimen subjected to cyclic loading. (a) Hits vs. loading cycles; (b) numerical derivative of hits vs. loading cycles. 
application of nonlocal damage models is impractical. Enrichment-based nonlocal formulations will be investigated to eliminate spurious macroscale mesh dependency without the need for high level of mesh resolution at the macroscale. Second, despite reasonable qualitative agreement of the model predictions with the experimental analysis of failure events with acoustic emission testing, a more detailed experimental analysis will provide accurate assessment of the predictive capability of the proposed multiscale computational framework. A detailed investigation based on image-based micromechanical analysis of failure events during cyclic loading will be conducted in the near future.

\section{Acknowledgements}

The authors gratefully acknowledge the financial support and experimental facilities provided by the Air Vehicles Directorate of the Air Force Research Laboratory (Contract No: F3446-09-46-SC0101 through General Dynamics Information Technology Inc.).

\section{References}

[1] R. A. A. Couillard and P. Schwartz. Bending fatigue of carbon-fiber-reinforced epoxy composite strands. Compos. Sci. Technol., 57:229-235, 1997.

[2] J. R. Gregory and S. M. Spearing. Constituent and composite quasi-static and fatigue fracture experiments. Compos. Part. A-Appl. S., 36:665-674, 2005.

[3] V. P. Nguyen, O. Lloberas-Valls, M. Stroeven, and L. J. Sluys. Computational homogenization for multiscale crack modeling implementational and computational aspects. Int. J. Numer. Meth. Eng., 2011.

[4] P. Raghavan and S. Ghosh. A continuum damage mechanics model for unidirectional composites undergoing interfacial debonding. Mech Mater, 37:955-979, 2005.

[5] T. B. Belytschko, S. Loehnert, and J. H. Song. Multiscale aggregating discontinuities: a method for circumventing loss of material stability. Int. J. Numer. Meth. Engng., 73:869-894, 2008.

[6] J. D. Hochhalter, D. J. Littlewood, Jr. Christ, R. J., M. G. Veilleux, J. E. Bozek, A. R. Ingraffea, and A. M. Maniatty. A geometric approach to modeling microstructurally small fatigue crack formation: Ii. physically based modeling of microstructure-dependent slip localization and actuation of the crack nucleation mechanism in aa 7075-t651. Modelling and Simulation In Materials Science and Engineering, 18(4), June 2010.

[7] R. Krueger and T. K. O'Brien. A shell/3d modeling technique for the analysis of delaminated composite laminates. Compos. Part. A-Appl. S., 32:25-44, 2001.

[8] S. Li, M. D. Thouless, A. M. Waas, J. A. Schroeder, and P. D. Zavattieri. Use of mode-I cohesivezone models to describe the fracture of an adhesively-bonded polymer-matrix composite. Compos. Sci. Technol., 65:281-293, 2005.

[9] D. B. P. Huynh and T. Belytschko. The extended finite element method for fracture in composite materials. Int. J. Numer. Meth. Eng., 77:214-239, 2009. 
[10] K. L. Reifsnider. The critical element model - a modeling philosophy. Eng. Fract. Mech., 25:739749, 1986.

[11] V. Tamuzs, K. Dzelzitis, and K. Reifsnider. Prediction of the cyclic durability of woven composite laminates. Compos. Sci. Technol., 68:2717-2721, 2008.

[12] N. V. Akshantala and R. Talreja. A micromechanics based model for predicting fatigue life of composite laminates. Mat. Sci. Eng. A-Struct., 285:303-313, 2000.

[13] M. M. Shokrieh and L. B. Lessard. Progressive fatigue damage modeling of composite materials, part I: Modeling. J. Compos. Mater., 34:1056-1080, 2000.

[14] S. M. Spearing, P. W. R. Beaumont, and M. F. Ashby. Fatigue damage mechanics of compositematerials .2. a damage growth-model. Compos. Sci. Technol., 44:169-177, 1992.

[15] J. M. Guedes and N. Kikuchi. Preprocessing and postprocessing for materials based on the homogenization method with adaptive finite element methods. Comput. Meth. Appl. Mech. Engng., 83:143-198, 1990.

[16] K. Terada and N. Kikuchi. Nonlinear homogenization method for practical applications. In S. Ghosh and M. Ostoja-Starzewski, editors, Computational Methods in Micromechanics, volume AMD-212/MD-62, pages 1-16. ASME, 1995.

[17] I. Babuska. Homogenization and application. mathematical and computational problems. In B. Hubbard, editor, Numerical Solution of Partial Differential Equations - III, SYNSPADE. Academic Press, 1975.

[18] A. Benssousan, J. L. Lions, and G. Papanicolaou. Asymptotic Analysis for Periodic Structures. North-Holland, Amsterdam, 1978.

[19] E. Sanchez-Palencia. Non-homogeneous media and vibration theory, volume 127 of Lecture Notes in Physics. Springer-Verlag, Berlin, 1980.

[20] P. M. Suquet. Elements of homogenization for inelastic solid mechanics. In E. Sanchez-Palencia and A. Zaoui, editors, Homogenization Techniques for Composite Media. Springer-Verlag, 1987.

[21] G. J. Dvorak. Transformation field analysis of inelastic composite materials. Proc. R. Soc. Lond. A, 437:311-327, 1992.

[22] J. Yvonnet and Q.-C. He. The reduced model multiscale method (R3M) for the non-linear homogenization of hyperelastic media at finite strains. J. Comput. Phys., 223:341-368, 2007.

[23] J. Fish, K. L. Shek, M. Pandheeradi, and M. S. Shephard. Computational plasticity for composite structures based on mathematical homogenization: Theory and practice. Comput. Meth. Appl. Mech. Engng., 148:53-73, 1997.

[24] J. Aboudi. A continuum theory for fiber-reinforced elastic-viscoplastic composites. J. Eng. Sci., 20(55):605-621, 1982.

[25] S. Ghosh and S. Moorthy. Elastic-plastic analysis of arbitrary heterogeneous materials with the voronoi cell finite element method. Comput. Methods Appl. Mech. Engng., 121(1-4):373-409, 1995. 
[26] H. Moulinec and P. Suquet. A fast numerical method for computing the linear and nonlinear properties of composites. C. R. Acad. Sc. Paris II, 318:1417-1423, 1994.

[27] C. Oskay and J. Fish. Eigendeformation-based reduced order homogenization for failure analysis of heterogeneous materials. Comp. Meth. Appl. Mech. Engng., 196(7):1216-1243, 2007.

[28] R. Crouch and C. Oskay. Symmetric meso-mechanical model for failure analysis of heterogeneous materials. Int. J. Mult. Comp. Eng., 8:447:461, 2010.

[29] M. H. J. W. Paas, P. J. G. Schreurs, and W. A. M. Brekelmans. A continuum approach to brittle and fatigue damage: Theory and numerical procedures. Int. J. Solids. Struct., 30:579-599, 1993.

[30] C. Oskay and J. Fish. Fatigue life prediction using 2-scale temporal asymptotic homogenization. Int. J. Numer. Meth. Engng., 61:329-359, 2004.

[31] C. Oskay and J. Fish. Multiscale modeling of fatigue for ductile materials. Int. J. Comp. Multiscale Engng., 2, 2004.

[32] J. Fish and C. Oskay. A nonlocal multiscale fatigue model. Mech. Adv. Materials and Structures, 12:485-500, 2005.

[33] J. Fish and Q. Yu. Computational mechanics of fatigue and life predictions for composite materials and structures. Comput. Meth. Appl. Mech. Engng., 191:4827-4849, 2002.

[34] E. Gal, Z. Yuan, W. Wu, and J. Fish. A multiscale design system for fatigue life prediction. Int. J. Multiscale Com., 5:435-446, 2007.

[35] R. L. Burden and J. D. Faires. Numerical Analysis. Brooks/Cole, Pacific Grove, CA, 6th edition, 1997.

[36] ASTM Standard D3039M-08. Standard test method for tensile properties of polymer matrix composite materials. Annual Book of ASTM Standards, 2008.

[37] ASTM Standard D3518M-94. Standard test method for in-plane shear response of polymer matrix composite materials by tensile test of a $+/-45^{\circ}$ laminate. Annual Book of ASTM Standards, 2007.

[38] ASTM Standard D3479M-96. Standard test method for tension-tension fatigue of polymer matrix composite materials. Annual Book of ASTM Standards, 2007.

[39] T. Breitzman, R. Lipton, and E. Iarve. Local field assessment inside multiscale composite architectures. Multiscale Model. Simul., pages 937-962, 2007. 